\title{
18. CHANGES IN THE DYNAMICS OF WESTERN EQUATORIAL ATLANTIC SURFACE CURRENTS AND BIOGENIC PRODUCTIVITY AT THE “MID-PLEISTOCENE REVOLUTION" ( 930 KA)
}

\author{
F.C. Bassinot, ${ }^{2}$ L. Beaufort ${ }^{3}$ E. Vincent,${ }^{3}$ and L. Labeyrie ${ }^{2}$
}

\begin{abstract}
We studied two sites cored during Ocean Drilling Program Leg 154 on the Ceara Rise in the western equatorial Atlantic: Sites 925 (3041 m water depth) and 928 (4012 m water depth). Both sites were sampled every $5 \mathrm{~cm}$ from about 25 to 38 meters below sea floor (mbsf; 680- to 1100-ka interval) for a high-resolution study of low-latitude climatic variations and changes in carbonate deposition associated with the first large-amplitude glaciation of the late Quaternary (isotopic Stage 22 at about 880 ka) and the subsequent development of the 100-k.y. glacial-interglacial oscillations (the "mid-Pleistocene revolution"). We measured carbonate and coarse fraction contents of samples from both sites. From Site 925 samples, we also measured $\delta^{18} \mathrm{O}$ of the planktonic species Globigerinoides ruber and the relative abundance of the calcareous nannofossil Florisphaera profunda, an index of past changes in nutricline depth (which permits us, by proxy, to reconstruct changes in thermocline dynamics).

Our results indicate that carbonate deposition was mainly controlled by changes in biogenic productivity and dilution by terrigenous inputs from the Amazon River. In the time interval studied, all of our paleoclimatic indicators show a dominant 41k.y. cyclicity. We suggest that 41-k.y. oscillations in the F. profunda record reflect changes in the thermocline depth resulting either from north-south oscillations of the Intertropical Convergence Zone or from changes in trade-wind intensity. These changes were most probably controlled by high-latitude variations in sea-surface temperatures that modified the subtropical gyre dynamics and were responding to changes in the volume of continental ice sheets. The "mid-Pleistocene revolution" appears to be associated with an important change in the equatorial Atlantic surface current dynamics as shown by the drastic change in the phase relationships between $1 / 41-\mathrm{k} . \mathrm{y}^{-1}$ filtered records that takes place over a short period of time centered roughly on $930 \mathrm{ka}$. This change may result from an important modification of the subtropical gyre dynamics at the initiation of the large Laurentide ice sheet. Before the "mid-Pleistocene revolution," 41-k.y. carbonate mass-accumulation rate cycles were out of phase with $F$. profunda cycles, indicating that biogenic productivity was most probably responding to changes in regional or global changes in nutrient content rather than to local changes in the nutricline (thermocline) dynamics. Starting at about 900 k.y., the biogenic productivity appears to be in phase with changes in the nutricline depth.
\end{abstract}

\section{INTRODUCTION}

Although 100-k.y climatic oscillations have been identified during other periods such as the Neogene (i.e., Beaufort and Aubry, 1990; Beaufort, 1994), they appear to be stronger during the late Pleistocene than during any other period. One of the major puzzles of the Ice Ages is the mid-Pleistocene onset of these large-amplitude 100-k.y. ice volume oscillations that dominated Earth's climate over the last 700 k.y. The exact timing and mechanisms of this onset are still a matter of debate. Some of the early studies that dealt with changes in dominant climatic periodicities favored a rapid transition at about 0.8-0.9 Ma (Shackleton and Opdyke, 1976; Pisias and Moore, 1981). However, recent studies concluded that the transition from 41-k.y. to 100-k.y. dominant oscillations has more probably been progressive, taking place approximately in the interval 1.0-1.2 to $0.6 \mathrm{Ma}$ (i.e., Ruddiman et al., 1989; Imbrie et al., 1993; Berger et al., 1994). By 0.9 Ma, the 100-k.y. power of climatic indicators from Deep Sea Drilling Project (DSDP) Site 607 in the North Atlantic approached or achieved a phase lock with orbital eccentricity for the first time, which suggests that the climate had really begun to develop the 100-k.y. power that dominates the late Pleistocene history (Ruddiman et al., 1989). Statistical detection of the mid-Pleistocene transition in mean and variance of Pleistocene $\delta^{18} \mathrm{O}$ records indicates that Earth's climatic system may have experienced an abrupt change around $0.9 \mathrm{Ma}$ that was characterized by an increase in ice mass and a global cooling (Maasch, 1988). It is not yet clear whether the rapid

'Shackleton, N.J., Curry, W.B., Richter, C., and Bralower, T.J. (Eds.), 1997. Proc. ODP, Sci. Results, 154: College Station, TX (Ocean Drilling Program).

${ }^{2}$ Centre des Faibles Radioactivités (CNRS/CEA), Domaine du CNRS, 91190 Gifsur-Yvette, France. Franck.Bassinot@cfr.cnrs-gif.fr

${ }^{3}$ Laboratoire de Géologie du Quaternaire, CNRS, CEREGE, BP 80, 13545 Aix-enProvence, France. and global change of the climate system that resulted in the first severe glaciation of the late Pleistocene (isotopic Stage 22) actually "triggered" the development of the 100-k.y. oscillations or if it corresponded to a "threshold jump" in a progressively evolving climatic system in which northern ice sheets had already initiated a new mode of oscillations. It appears well established, however, that the existence of large northern hemisphere ice sheets is an essential condition for developing the "saw-tooth" $100-\mathrm{k} . \mathrm{y}$. cycles typical of the late Pleistocene (e.g., Saltzman, 1987; Ghil and Le Treut, 1981; Le Treut and Ghil, 1983; DeBlonde and Peltier, 1991; Imbrie and Imbrie, 1980; and review in Imbrie et al., 1993). This conclusion, mostly derived from a nonlinear modeling approach, has been strongly supported by available paleoclimatic data. At ODP Site 677, for instance, the variance increase in the $1 / 100-\mathrm{k} . \mathrm{y}^{-1}$ band, which took place between about 1.0 and $0.7 \mathrm{Ma}$, occurred mainly as an enrichment of $\delta^{18} \mathrm{O}$ values during glacial stages and was recorded both in planktonic and benthic foraminifers, thus suggesting a progressive increase in the size of the northern hemisphere ice sheets (Imbrie et al., 1993). The equatorward shift of the polar front associated with the growth of these large ice sheets is also reflected in the southward progression of ice-rafted detritus deposited in the North Atlantic (Ruddiman, McIntyre, et al., 1986).

The major change of the climate system at about $0.9 \mathrm{Ma}$ (the "mid-Pleistocene revolution," Berger et al., 1994) is still poorly understood. Ruddiman, Raymo, et al. (1986) and Ruddiman and Kutzbach (1989) have suggested that the development of large amplitude glaciations in the mid-Pleistocene could have been triggered by changes in the elevation of the Sierra Nevada and the Tibetan Plateau, which would have affected tropospheric circulation and resulted in cooling over North America and Europe. This scenario seems to explain the long-term evolution of the climate system during the Pliocene-Pleistocene. The abruptness of the superimposed climatic jump at about 0.9 Ma points towards a "threshold" response of the 
climatic system that involves elements with a rapid time response, such as oceanic circulation. The evolution of Atlantic-Pacific $\delta^{13} \mathrm{C}$ gradients reconstructed by Raymo et al. (1990) shows that glacial episodes corresponding to isotopic Stages 24 and 22 were the first glacial episodes of the late Pleistocene, which are characterized by a clear reduction of the North Atlantic Deep Water (NADW) production. The convergence of $\delta^{13} \mathrm{C}$ values between the deep Atlantic and Pacific during isotopic Stages 24 and 22 indicates that these times not only experienced a drastic reduction of NADW production, but also probably experienced a fundamentally different pattern of circulation than those observed during later glacial stages of the late Pleistocene, with production of nutrient-depleted bottom water in the Pacific and/ or increased residence time of deep water in the Atlantic (Raymo et al., 1990).

Given these observations, the Ceara Rise area appears to be a zone of extreme interest and may provide more information about the mechanisms involved in the "mid-Pleistocene revolution." Today, this area is characterized by a south-north cross-equatorial transport of upper waters, which bring heat and salt gained in the tropics to the North Atlantic and play a crucial role in the "Great Conveyor Belt" circulation as the main branch of the return flow of the North Atlantic Deep Water (NADW) thermohaline cell. Of the $13 \mathrm{~Sv}$ that are exported southward as NADW and are replaced by southern ocean water, about $7 \mathrm{~Sv}$ are supplied by northern cross-equatorial transport of warm $\left(>24^{\circ} \mathrm{C}\right.$ ), near-surface water (upper 50-100 m) that travels along the equator as the South Equatorial Current (SEC), is deflected northward along the coast of Brazil, and feeds the Gulf Stream and the North Atlantic Drift after travel through the Caribbean (Schmitz and McCartney, 1993). Along the equator, this upper water flow is under the direct control of wind-driven circulation. Today, both the thermal equator and the Intertropical Convergence Zone (ITCZ) are deflected to the north of the geographic equator in the western equatorial Atlantic. Past changes in the geographical position of these important boundaries could have had an important impact on the north Atlantic thermohaline cell and/or on the northern hemisphere ice sheet build-up by modifying the cross-equatorial water, salt, and heat transport to the North Atlantic. It appears, therefore, extremely important to clearly understand past changes in western equatorial Atlantic hydrologic conditions considering the key role of this area in the transfer of energy and water to the North Atlantic.

The relative abundance of the coccolithophorid species Florisphaera profunda has been used as a powerful indicator for reconstructing past changes in the thermocline dynamics of the eastern equatorial Atlantic (Molfino and McIntyre, 1990). F. profunda is one of the rare coccolithophorid species preserved in the fossil record that is restricted to the lower euphotic zone (Okada and Honjo, 1973). The conceptual model proposed by Molfino and McIntyre (1990) associates high abundances of $F$. profunda with a deep nutricline and, by proxy, a deep thermocline (weak divergence), and low abundances of $F$. profunda with a shallow nutricline and, by proxy, a shallow thermocline (strong divergence). The study of $F$. profunda abundance in sediments cored on Ceara Rise during Leg 154 offers a unique opportunity to gain some important insights on past changes of the winddriven equatorial dynamics at the time of the "mid-Pleistocene revolution."

\section{STUDY AREA AND SITE PRESENTATION}

Sites 925 and 928 were cored during Leg 154 on the Ceara Rise (western equatorial Atlantic), a bathymetric high that formed at 80 $\mathrm{Ma}$ and is draped with a thick sequence of lithogenic (mostly input from the Amazon River) and biogenic sediments (Fig. 1). The rise is located beneath a warm surface-water pool that exhibits low productivity. The CLIMAP reconstruction has shown that this warm-water pool experienced only a small temperature decrease $\left(<2^{\circ} \mathrm{C}\right)$ at the
Last Glacial Maximum (CLIMAP, 1981). The Ceara Rise is bathed by NADW and Antarctic Bottom Water. The present mixing zone between these two deep water masses is located between 4000 and 4500 $\mathrm{m}$, and corresponds to a steep gradient in deep-water chemistry that controls the dissolution of calcium carbonate (Curry and Lohmann, 1990).

Site 925 , located at $4^{\circ} 12.249^{\prime} \mathrm{N}, 43^{\circ} 29.334^{\prime} \mathrm{W}$, is the shallowest site cored during Leg 154 at $3041 \mathrm{~m}$ of water depth (Fig. 1). It is situated well above the present lysocline in this area, which is found at about 4000-4300 m (Broecker and Takahashi, 1978; Thunell, 1982a). Pelagic sediments from Site 925 have experienced little dissolution during the Cenozoic, as demonstrated by the usually very good preservation of calcareous microfossils observed during Leg 154 (see Biostratigraphy section, Shipboard Scientific Party, 1995a).

Site $928\left(5^{\circ} 27.320^{\prime} \mathrm{N} 43^{\circ} 44.884^{\prime} \mathrm{W}\right)$ is located at $4012 \mathrm{~m}$ of water depth, about $100 \mathrm{~m}$ above the present depth of the lysocline. This position makes Site 928 extremely sensitive to past changes in the production rate of NADW that resulted in vertical migration of the mixing zone and changes in carbonate chemistry.

\section{COMPOSITE DEPTHS AND MATERIAL}

To correct for sediment gaps and disturbances at core breaks (Ruddiman et al., 1987), continuous sedimentary records at Sites 925 and 928 were obtained by splicing intervals from adjacent holes. Splice tie-points were determined during Leg 154 based on highresolution $(3-5 \mathrm{~cm}$ intervals) physical properties records (magnetic susceptibility, color reflectance) obtained on board the JOIDES Resolution. These tie-points are presented in the "Composite Section" sections of Shipboard Scientific Party (1995a, 1995b). All depths below seafloor are expressed in meters composite depth (mcd) hereafter.

For our study of the "mid-Pleistocene revolution," we decided to focus on the stratigraphic interval ranging from isotopic Stage 17 to isotopic Stage 32 (spanning roughly the time interval 680-1100 ka). This interval has been identified on Sites 925 and 928 based on the color reflectance and magnetic susceptibility profiles as well as on biostratigraphy events recognized on board the JOIDES Resolution such as the reappearance of the medium Gephyrocapsa species that occurred around isotopic Stage 27 in the Atlantic Ocean (Raffi et al., 1993). This datum occurs at $34.40 \mathrm{mcd}$ at Site 925 and at $35.18 \mathrm{mcd}$ at Site 928 (see "Biostratigraphy" sections, Shipboard Scientific Party, 1995a, 1995b). Based on this preliminary identification of the interval of interest, the sedimentary records were sampled at $5-\mathrm{cm}$ intervals from 25.85 to $38 \mathrm{mcd}$ at Site 925 (splice from Holes $925 \mathrm{C}$ and 925D) and from 23.25 to $42 \mathrm{mcd}$ at Site 928 (splice from Holes 928A, $928 \mathrm{~B}$, and $928 \mathrm{C}$ ). The 5-cm sampling interval is equivalent to a time interval of about $1.7 \mathrm{k} . \mathrm{y}$. at both sites. Carbonate and sand-fraction contents $(>63 \mu \mathrm{m})$ were measured on samples from Sites 925 and 928. Oxygen $\left(\delta^{18} \mathrm{O}\right)$ and carbon $\left(\delta^{13} \mathrm{C}\right)$ isotopic compositions of the planktonic foraminifer Globigerinoides ruber and the relative abundance of the calcareous nannofossil Florisphaera profunda were measured in samples from Site 925.

\section{METHODS Carbonate Content}

Weight percentages of calcium carbonate in dry bulk samples were measured at the Laboratoire de Géologie du Quaternaire (LGQ, Marseille, France) using a modified "Bernard calcimeter," which measures the volume of $\mathrm{CO}_{2}$ released at atmospheric pressure when the sample is reacted with $\mathrm{HCl}-1 \mathrm{~N}$. The accuracy of the method is within $\pm 2 \%-3 \%$ (estimated from 50 measurements of standards with known $\mathrm{CaCO}_{3}$ content ranging between $50 \%$ and $100 \%$ ). 


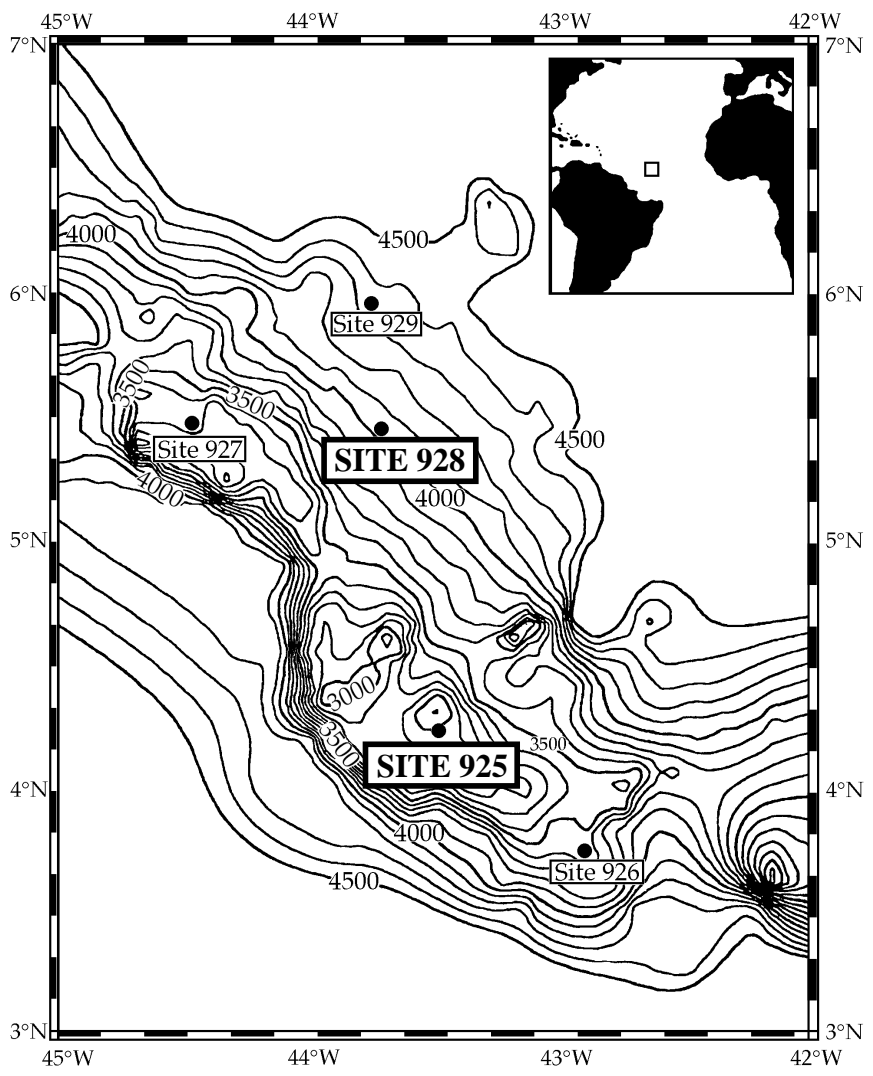

Figure 1. Location of Sites 925 and 928 cored during Leg 154 on Ceara Rise.

\section{Coarse Fraction Content}

The sand-size fraction was obtained by wet-sieving samples using a $63-\mu \mathrm{m}$ mesh sieve. The dry, greater than $63-\mu \mathrm{m}$ fraction was then weighed to calculate the percentage of sand fraction. In Site 925 samples, the sand-size fraction was subsieved using 250 - and $355-\mu \mathrm{m}$ mesh sieve to isolate large foraminifer shells for stable isotope studies (see below).

\section{$\delta^{18} \mathrm{O}$ and $\delta^{13} \mathrm{C}$ Measurements}

Stable isotopic measurements were performed on the planktonic species Globigerinoides ruber picked in the narrow size range 250$355 \mu \mathrm{m}$ to avoid, as much as possible, $\delta^{13} \mathrm{C}$ variations related to ontogenetic evolution (e.g., Berger et al., 1978; Duplessy et al., 1981). Samples were roasted at $400^{\circ} \mathrm{C}$ prior to analysis to remove organic matter. Foraminifers were analyzed at the Centre des Faibles Radioactivités (CFR, Gif-sur-Yvette, France) using a VG-Optima mass spectrometer equipped with a carbonate device. Samples of about $100 \mu \mathrm{g}(\sim 10$ shells of $G$. ruber $)$ were reacted with phosphoric acid at $90^{\circ} \mathrm{C}$ to produce $\mathrm{CO}_{2}$, which was analyzed in the mass spectrometer. Following the conventional notation, results are expressed in $\delta$ with respect to the Pee Dee Belemnite standard. Analytical precision, based on replicate measurements of the NBS19 standard, is \pm 0.06 for $\delta^{18} \mathrm{O}$ and \pm 0.02 for $\delta^{13} \mathrm{C}$.

\section{Florisphaera profunda}

Relative abundances of the calcareous nannofossil Florisphaera profunda were measured on smear slides using a light microscope at the LGQ. At least two hundred coccoliths per slide were counted for the calculation of the percentage of $F$. profunda. The $95 \%$ confidence interval varies between $\pm 2 \%$ and $\pm 7 \%$ depending on the percentage and the amount of coccoliths counted (Mosimann, 1965).

\section{Mass-Accumulation Rates}

Mass-accumulation rates (MAR) of the carbonate and noncarbonate fraction were calculated according to the classical formulae:

$$
\begin{gathered}
\mathrm{MAR}_{\text {carb }}=D B D \times F_{\text {carb }} \times S R \\
\mathrm{MAR}_{\text {non-carb }}=D B D \times\left(1-F_{\text {carb }}\right) \times S R
\end{gathered}
$$

where $D B D$ is the dry-bulk density (in $\mathrm{g} / \mathrm{cm}^{3}$ ), $F_{\text {carb }}$ is the dry-weight fraction of carbonate in the sediment, $S R$ is the sedimentation rate (cm/k.y.).

Dry-bulk densities were obtained on board the JOIDES Resolution from measurements of the wet and dry sediment masses (obtained with a precision of $\pm 0.01 \mathrm{~g}$ using a Scitech electronic balance) and the wet sediment volumes (obtained using a Quantachrome pycnometer with a precision of $\pm 0.02 \mathrm{~cm}^{3}$; for details, see "Physical Properties" sections, Curry, Shackleton, Richter, et al. [1995]).

The discrete dry-bulk density data sets from Sites 925 and 928 have a low sampling resolution (13 values for our interval of interest from Site 925 and 10 values from Site 928) and do not permit us to address changes that might be associated with glacial-interglacial variations in sediment composition. To determine the effect of such climate-induced dry-bulk density changes on the calculation of massaccumulation rates, we conducted a short, high-resolution analysis of index properties variations based on the wet-bulk density record obtained on board the JOIDES Resolution at 2-cm spacing intervals with the Gamma Ray Attenuation Porosity Evaluator. Our results showed that bulk density shows low-amplitude glacial-interglacial variations. Thus, MARs appear to be dominated by changes in carbonate contents (or noncarbonate contents), which undergo largeamplitude variations (about $30 \%$ of their average value at Site 925 and $44 \%$ at Site 928 ) and do not depend on small-amplitude changes of index properties (which produce variations of about $2 \%$ and $4.5 \%$ of their average value from Sites 925 and 928, respectively).

Data from Sites 925 and 928 are presented in Tables 1 and 2, and plotted vs. depth in Figures 2 and 3.

\section{AGE MODEL}

The detailed isotopic record obtained from Site 925 extends from isotopic Stages 17 to 32 (685-1100 ka; Fig. 2). It shows distinctly all the isotopic oscillations characteristic of this time interval, such as the three precession-related oscillations of Stage 21 that have been recently observed in other low-latitude isotopic records (Shackleton et al., 1990; Bassinot et al., 1994b). An age model has been developed at Site 925 by tuning the oxygen isotopic curve obtained on G. ruber to the ice-volume model of Imbrie and Imbrie (1980). This model assumes that the rate of the climate response (growth or decay of ice sheets) is proportional to the magnitude of the summer insolation forcing at $65^{\circ} \mathrm{N}$. The model has been calculated using the monthly insolation series for July at $65^{\circ} \mathrm{N}$ of Berger and Loutre (1991). The resulting curve has proved to be a powerful tuning target for the late Pleistocene (Martinson et al., 1987; Bassinot et al., 1994b) as well as for the early Pleistocene (Shackleton et al., 1990).

Because our climatic series do not extend continuously to the present, we had to choose an initial starting point for our tuning procedure. As an initial tie-point, we correlated isotopic Event 20.2 (at about $29 \mathrm{mcd}$ ) to the minimum in the ice-volume model found at about $790 \mathrm{ka}$. Because Stage 20.2 is a well-defined $\delta^{18} \mathrm{O}$ event situated just below the Brunhes-Matuyama magnetic reversal (which oc- 
curs in Stage 19), special attention has been given during the last five years to its precise dating through orbital tuning. We chose our depthage correlation for Stage 20.2 according to the tuning solution proposed by Shackleton et al. (1990) for ODP Site 677, recently confirmed by the detailed astronomical tuning of the continuous 900-k.y. isotopic record from giant piston Core MD900963 (Bassinot et al., $1994 b$ ) in which good magnetostratigraphy was available. Subsequent correlations of the isotopic record of Site 925 to the ice-volume model were performed using the "linage" subroutine of the program "analyseries" developed at the CFR (Paillard and Labeyrie, 1995). The tuning approach employed an iterative procedure. After depthto-age control points were chosen, ages of the entire $\delta^{18} \mathrm{O}$ series were linearly interpolated and the resulting time series was filtered at $1 / 21$ and $1 / 41 \mathrm{k} . \mathrm{y}^{-1}$ to extract the precession and obliquity components. These filtered series were then compared to the 1/21-k.y. ${ }^{-1}$ and the $1 /$ 41-k.y. ${ }^{-1}$ components of the ice-volume model. Age-control points were modified in intervals where important mismatches were observed between the filtered isotope record and the filtered ice volume curve. The corrected time series was filtered again for comparison. The iterative procedure was repeated until a good coherency $(>0.9)$ was achieved between the $1 / 21$ and $1 / 41-\mathrm{k} . \mathrm{y} .^{-1}$ filters obtained from the isotopic record and the ice-volume model (Fig. 4). As an additional check, the isotopic record is also compared with the isotopic record from ODP Site 677 (Shackleton et al., 1990).

Final control points of our age model are given in Table 3. The isotopic record from Site 925 spans the time interval from about 680 to $1100 \mathrm{ka}$. The orbital chronology developed for Site 925 was transferred to Site 928 by correlation of the carbonate and coarse-fraction records (Figs. 5, 6).

\section{RESULTS}

\section{Carbonate and Noncarbonate Sedimentation}

The mean value of the sand fraction content is $13.3 \%$ (standard deviation $1 \sigma=6.1 \%)$ at Site 925 and $6.6 \%(\sigma=6.3 \%)$ at Site 928 . Carbonate contents average $41.9 \%(\sigma=12.4 \%)$ at Site 925 and $28.8 \%(\sigma=13.3 \%)$ at Site 928 (Figs. 5, 6). Visual observation with a binocular microscope showed that the sand fraction at Sites 925 and 928 consists entirely of foraminifer shells. These foraminifers appear to be particularly well preserved at the shallower Site 925, which is located at $3041 \mathrm{~m}$ of water depth, about $1000 \mathrm{~m}$ above the present lysocline. This good preservation state of foraminifer shells indicates that Site 925 remained in a supralysoclinal position during most of the time interval studied, and suggests that dissolution was not a major factor controlling biogenous carbonate deposition. Site 928 is located at $4012 \mathrm{~m}$ of water depth (about $100 \mathrm{~m}$ above the present lysocline in this area). Lower mean values of carbonate content and grain size measured at Site 928 than at Site 925 probably result from enhanced dissolution of carbonate at the deeper site. With increasing dissolution, sand fraction content is reduced by the progressive breakdown of weakened foraminifer shells into small fragments (Thunell, 1982b; Berger et al., 1982; Peterson and Prell, 1985; Le and Shackleton, 1992; Bassinot et al., 1994a).

At Sites 925 and 928, the carbonate and coarse-fraction records show in-phase, large-amplitude oscillations (Figs. 5, 6). Carbonate and sand fraction contents appear to be positively correlated with linear regression coefficients $\left(\mathrm{R}^{2}\right)$ around 0.7 at both sites (Fig. 7). The good preservation of foraminifer shells indicates that dissolution changes are unlikely to drive such large-amplitude changes in carbonate and coarse fraction contents at Site 925. The deeper Site 928 is more affected by dissolution, as shown by the poorer state of preservation of foraminifer shells. However, a few lines of evidence suggest that dissolution may not have affected carbonate content at Site 928 either. First, changes in carbonate and coarse fraction content at Site 928 appear to mimic changes observed in Site 925 and this im- plies, therefore, that it is probably the same main mechanisms that act at both sites. Furthermore, if dissolution was the main factor driving large-amplitude changes observed at Site 928, we would expect to see a higher variance in the coarse-fraction record than in the carbonate record, because numerous studies of pelagic calcareous sediments have shown that foraminifer fragmentation (and, thus, coarse fraction) is much more sensitive to dissolution changes than is carbonate content (e.g., Peterson and Prell, 1985; Bassinot et al., 1994a). The fact that coarse-fraction variations at Site 928 show a relatively low range of variability $(\sigma=6.6 \%)$ compared to carbonate-content variations $(\sigma=13.3 \%)$ seems to indicate, therefore, that dissolution does not play a major role in controlling large-amplitude carbonate content oscillations at Site 928. Although it is clear that additional analyses will be necessary to explore in detail the effects of dissolution on the composition of Ceara Rise sediments, we consider at this stage of our work that dissolution is unlikely to play an important role in carbonate content variations at Sites 925 and 928.

Preliminary studies performed on board the JOIDES Resolution showed that terrigenous material deposited upon the Ceara Rise consists primarily of fine-grained, noncalcareous particles (clay minerals) probably supplied by the Amazon River. Thus, large-amplitude variations observed in carbonate and coarse-fraction records from Sites 925 and 928 could reflect productivity and/or dilution changes that controlled the relative contribution of the coarse (foraminiferrich), calcareous biogenic material and the fine, noncalcareous terrigenous material to the bulk sediment. MARs of carbonate and noncarbonate material show that both dilution and productivity changes have played a role in controlling sedimentation at Ceara Rise in the 680- to 1100-ka time interval (Fig. 8). High carbonate-accumulation rates (high biogenic productivity) appear to be associated usually with interglacial/warm periods whereas high noncarbonate-accumulation rates are generally associated with glacial/cold periods. Changes in noncarbonate MARs may reflect changes in the terrigenous supply that are either associated with changes in climatic conditions over South America or that result, more likely, from sea level changes that modified the river erosion profiles and/or the sedimentation processes on the continental shelves and the Amazon Fan.

Our findings are in relatively good agreement with results from Mienert et al. (1988) concerning the late Quaternary sedimentological and physical properties changes at Ceara Rise (derived from the study of piston cores). These authors have shown that acoustic properties of Ceara Rise sediments over the last 250 k.y. reflect changes in the production of planktonic foraminifers. It is only below $4300 \mathrm{~m}$ ( lysocline depth) that large downcore, productivity-related $P$-wave velocity fluctuations gradually disappear because of dissolution of carbonate sediments.

If sedimentation on Ceara Rise were not affected by downslope movement or lateral advection of fine sediments by bottom currents, then the difference in carbonate accumulation rates between Sites 925 and $928\left(\Delta \mathrm{MAR}_{\mathrm{CaCO}_{3}}\right)$ could give us an indication of past changes in the dissolution gradient along the Ceara Rise. However, it appears that large-scale features observed in the $\triangle \mathrm{MAR}_{\mathrm{CaCO}_{3}}$ curve mimic relatively closely the changes observed in the $\triangle \mathrm{MAR}$ non- $\mathrm{CaCO}_{3}$ curve (obtained by subtracting the noncarbonate MAR curve at Site 928 from that at Site 925). The fact that both MAR gradients (carbonate and noncarbonate) show some covariance could suggest that reworking by bottom currents might have affected the sedimentation on the Ceara Rise.

\section{Changes in Florisphaera profunda Abundance}

The record of relative Florisphaera profunda abundance shows large-amplitude changes with a long-term oscillation superimposed on meter-scale variations. The curve shows an important minimum around isotopic Stages 27-25 followed by a striking maximum at the first severe late Pleistocene glaciation, isotopic Stage 22 (Fig. 5). 
Table 1. $\delta^{18} \mathrm{O}$ of Globigerinoides ruber, carbonate content, sand-sized fraction content, and Florisphaera profunda relative abundance measured from Site 925 samples.

\begin{tabular}{|c|c|c|c|c|c|c|c|c|c|c|}
\hline $\begin{array}{l}\text { Hole, core, section } \\
\text { interval }(\mathrm{cm})\end{array}$ & $\begin{array}{l}\text { Depth } \\
\text { (mcd) }\end{array}$ & $\begin{array}{c}\mathrm{CaCO}_{3} \\
(\%)\end{array}$ & $\begin{array}{c}>63 \mu \mathrm{m} \\
(\%)\end{array}$ & $\begin{array}{l}\delta^{18} \mathrm{O} \\
(\%)\end{array}$ & $\begin{array}{l}\delta^{13} \mathrm{C} \\
(\%)\end{array}$ & $\begin{array}{c}F . \\
\text { profunda } \\
(\%)\end{array}$ & $\begin{array}{l}\text { Hole, core, section } \\
\text { interval }(\mathrm{cm})\end{array}$ & $\begin{array}{l}\text { Depth } \\
\text { (mcd) }\end{array}$ & $\begin{array}{c}\mathrm{CaCO}_{3} \\
(\%)\end{array}$ & $\begin{array}{c}>63 \mu \mathrm{m} \\
(\%)\end{array}$ \\
\hline $925 \mathrm{C}-3 \mathrm{H}-5,128-129$ & 25.11 & & 7.3 & -0.71 & 1.05 & & $925 \mathrm{D}-3 \mathrm{H}-4,83-85$ & 30.09 & 44.6 & 10.0 \\
\hline 925D-3H-1, 113-115 & 25.89 & 30.7 & 5.9 & -1.26 & 1.36 & 22.8 & $925 \mathrm{D}-3 \mathrm{H}-4,88-90$ & 30.14 & 44.3 & 11.7 \\
\hline $925 \mathrm{D}-3 \mathrm{H}-1,118-120$ & 25.94 & 25.3 & 9.2 & -1.69 & 1.49 & & $925 \mathrm{D}-3 \mathrm{H}-4,93-95$ & 30.19 & 40.7 & 11.9 \\
\hline $925 \mathrm{D}-3 \mathrm{H}-1,123-125$ & 25.99 & 32.6 & 7.7 & -1.36 & 1.51 & 50.5 & $925 \mathrm{D}-3 \mathrm{H}-4,98-100$ & 30.24 & 36.9 & 13.7 \\
\hline $925 \mathrm{D}-3 \mathrm{H}-1,128-130$ & 26.04 & 57.7 & 17.4 & -1.61 & 1.68 & & $925 \mathrm{D}-3 \mathrm{H}-4,103-105$ & 30.29 & 23.7 & 6.1 \\
\hline $925 \mathrm{D}-3 \mathrm{H}-1,133-135$ & 26.09 & 69.9 & 23.3 & -1.77 & 1.48 & 47.6 & $925 \mathrm{D}-3 \mathrm{H}-4,108-110$ & 30.34 & 31.0 & 6.6 \\
\hline $925 \mathrm{D}-3 \mathrm{H}-1,138-140$ & 26.14 & 73.3 & 27.7 & -1.84 & 1.27 & 47.0 & $925 \mathrm{D}-3 \mathrm{H}-4,113-115$ & 30.39 & 49.4 & 15.3 \\
\hline $925 \mathrm{D}-3 \mathrm{H}-1,143-145$ & 26.19 & 70.8 & 22.3 & -1.84 & 1.27 & 42.9 & $925 \mathrm{D}-3 \mathrm{H}-4,118-120$ & 30.44 & 47.7 & 18.1 \\
\hline $925 \mathrm{D}-3 \mathrm{H}-1,148-150$ & 26.24 & 60.2 & 17.3 & -1.49 & 1.24 & & $925 \mathrm{D}-3 \mathrm{H}-4,123-125$ & 30.49 & 48.7 & 17.6 \\
\hline $925 \mathrm{D}-3 \mathrm{H}-2,3-5$ & 26.29 & 56.2 & 15.0 & -1.46 & 1.26 & 45.7 & $925 \mathrm{D}-3 \mathrm{H}-4,128-130$ & 30.54 & 54.0 & 15.7 \\
\hline $925 \mathrm{D}-3 \mathrm{H}-2,8-10$ & 26.34 & 51.4 & 17.3 & -1.52 & 1.10 & & $925 \mathrm{D}-3 \mathrm{H}-4,133-135$ & 30.59 & 42.3 & 16.8 \\
\hline $925 \mathrm{D}-3 \mathrm{H}-2,13-15$ & 26.39 & 54.0 & 18.6 & -1.68 & 1.03 & 14.4 & & 30.59 & & \\
\hline $925 \mathrm{D}-3 \mathrm{H}-2,18-20$ & 26.44 & 53.1 & 19.3 & -1.45 & 1.16 & & $925 \mathrm{D}-3 \mathrm{H}-4,138-140$ & 30.64 & 35.2 & 13.0 \\
\hline $925 \mathrm{D}-3 \mathrm{H}-2,23-25$ & 26.49 & 50.9 & 18.6 & -1.63 & 1.24 & 41.2 & $925 \mathrm{D}-3 \mathrm{H}-4,143-145$ & 30.69 & 30.1 & 12.1 \\
\hline $925 \mathrm{D}-3 \mathrm{H}-2,28-30$ & 26.54 & 50.3 & 22.5 & -1.43 & 1.13 & & $925 \mathrm{D}-3 \mathrm{H}-4,148-150$ & 30.74 & 35.5 & 11.2 \\
\hline $925 \mathrm{D}-3 \mathrm{H}-2,33-35$ & 26.59 & 57.5 & 19.3 & -1.31 & 1.21 & 60.6 & $925 \mathrm{D}-3 \mathrm{H}-5,3-5$ & 30.79 & 48.7 & 14.5 \\
\hline $925 \mathrm{D}-3 \mathrm{H}-2,38-40$ & 26.64 & 50.3 & 23.5 & -1.10 & 0.95 & & $925 \mathrm{D}-3 \mathrm{H}-5,8-10$ & 30.84 & 58.0 & 20.1 \\
\hline $925 \mathrm{D}-3 \mathrm{H}-2,43-45$ & 26.69 & 47.8 & 19.0 & -0.63 & 1.17 & 59.2 & & 30.84 & & \\
\hline $925 \mathrm{D}-3 \mathrm{H}-2,48-50$ & 26.74 & 35.5 & 10.8 & -0.55 & 0.61 & & $925 \mathrm{D}-3 \mathrm{H}-5,13-15$ & 30.89 & 70.5 & 27.9 \\
\hline $925 \mathrm{D}-3 \mathrm{H}-2,53-55$ & 26.79 & 31.4 & 9.6 & -0.65 & 0.70 & 83.5 & $925 \mathrm{D}-3 \mathrm{H}-5,18-20$ & 30.94 & 67.6 & 28.9 \\
\hline $925 \mathrm{D}-3 \mathrm{H}-2,58-60$ & 26.84 & 31.1 & 9.6 & -0.61 & 0.63 & & & 30.94 & & 2. \\
\hline 925D-3H-2, 63-65 & 26.89 & 30.1 & 5.2 & -0.36 & 0.66 & 66.2 & 925D-3H-5, 23-25 & 30.99 & 54.0 & 26.3 \\
\hline $925 \mathrm{D}-3 \mathrm{H}-2,68-70$ & 26.94 & 31.3 & 9.1 & -0.52 & 1.16 & & $925 \mathrm{D}-3 \mathrm{H}-5,28-30$ & 31.04 & 50.0 & 16.4 \\
\hline $925 \mathrm{D}-3 \mathrm{H}-2,73-75$ & 26.99 & 37.9 & 9.1 & -1.13 & 1.08 & 33.1 & $925 \mathrm{D}-3 \mathrm{H}-5,33-35$ & 31.09 & 37.5 & 16.1 \\
\hline $925 \mathrm{D}-3 \mathrm{H}-2,78-80$ & 27.04 & 40.1 & 13.8 & -1.13 & 0.89 & & $925 \mathrm{D}-3 \mathrm{H}-5,38-40$ & 31.14 & 33.0 & 11.2 \\
\hline $925 \mathrm{D}-3 \mathrm{H}-2,83-85$ & 27.09 & 47.2 & 13.3 & -0.91 & 0.92 & 29.2 & $925 \mathrm{D}-3 \mathrm{H}-5,43-45$ & 31.19 & 22.4 & 6.8 \\
\hline $925 \mathrm{D}-3 \mathrm{H}-2,88-90$ & 27.14 & 50.9 & 16.7 & -1.11 & 0.84 & & $925 \mathrm{D}-3 \mathrm{H}-5,48-50$ & 31.24 & 24.4 & 9.1 \\
\hline $925 \mathrm{D}-3 \mathrm{H}-2,93-95$ & 27.19 & 53.7 & 18.0 & -1.29 & 1.17 & 17.0 & $925 \mathrm{D}-3 \mathrm{H}-5,53-55$ & 31.29 & 26.0 & 8.0 \\
\hline $925 \mathrm{D}-3 \mathrm{H}-2,98-100$ & 27.24 & 52.8 & 16.4 & -1.14 & 1.14 & & $925 \mathrm{D}-3 \mathrm{H}-5,58-60$ & 31.34 & 27.0 & 8.1 \\
\hline $925 \mathrm{D}-3 \mathrm{H}-2,103-105$ & 27.29 & 57.1 & 20.4 & -1.36 & 1.59 & 22.9 & $925 \mathrm{D}-3 \mathrm{H}-5,63-65$ & 31.39 & 26.3 & 9.6 \\
\hline $925 \mathrm{D}-3 \mathrm{H}-2,108-110$ & 27.34 & 52.6 & 19.9 & -1.16 & 0.35 & & $925 \mathrm{D}-3 \mathrm{H}-5,68-70$ & 31.44 & 32.0 & 10.8 \\
\hline $925 \mathrm{D}-3 \mathrm{H}-2,113-115$ & 27.39 & 52.5 & 15.5 & -1.36 & 0.99 & 16.2 & $925 \mathrm{D}-3 \mathrm{H}-5,73-75$ & 31.49 & 33.1 & 11.5 \\
\hline $925 \mathrm{D}-3 \mathrm{H}-2,118-120$ & 27.44 & 50.3 & 17.2 & -1.20 & 0.54 & & $925 \mathrm{D}-3 \mathrm{H}-5,78-80$ & 31.54 & 35.0 & 10.5 \\
\hline $925 \mathrm{D}-3 \mathrm{H}-2,123-125$ & 27.49 & 49.1 & 15.9 & -1.19 & 0.99 & 29.0 & $925 \mathrm{D}-3 \mathrm{H}-5,83-85$ & 31.59 & 29.0 & 9.7 \\
\hline $925 \mathrm{D}-3 \mathrm{H}-2,128-130$ & 27.54 & 45.2 & 15.6 & -1.20 & 0.99 & & 925D-3H-5, 88-90 & 31.64 & 26.9 & 8.7 \\
\hline $925 \mathrm{D}-3 \mathrm{H}-2,133-135$ & 27.59 & 40.1 & 13.3 & -1.11 & 1.54 & 23.7 & $925 \mathrm{D}-3 \mathrm{H}-5,93-95$ & 31.69 & 24.9 & 9.3 \\
\hline $925 \mathrm{D}-3 \mathrm{H}-2,138-140$ & 27.64 & 29.3 & 9.1 & -0.85 & 0.65 & & $925 \mathrm{D}-3 \mathrm{H}-5,98-100$ & 31.74 & 28.9 & 10.1 \\
\hline $925 \mathrm{D}-3 \mathrm{H}-2,143-145$ & 27.69 & 26.3 & 5.0 & -0.63 & 0.91 & 36.6 & $925 \mathrm{D}-3 \mathrm{H}-5,103-105$ & 31.79 & 32.5 & 11.1 \\
\hline $925 \mathrm{D}-3 \mathrm{H}-2,148-150$ & 27.74 & 30.4 & 21.5 & -0.95 & 0.86 & 17.5 & $925 \mathrm{D}-3 \mathrm{H}-5,108-110$ & 31.84 & 43.9 & 13.4 \\
\hline $925 \mathrm{D}-3 \mathrm{H}-3,3-5$ & 27.79 & 29.2 & 7.6 & -0.82 & 0.65 & 39.0 & $925 \mathrm{D}-3 \mathrm{H}-5,113-115$ & 31.89 & 47.3 & 15.6 \\
\hline $925 \mathrm{D}-3 \mathrm{H}-3,8-10$ & 27.84 & 32.4 & 8.2 & -0.72 & 0.85 & & 925D-3H-5, 118-120 & 31.94 & 51.4 & 18.0 \\
\hline $925 \mathrm{D}-3 \mathrm{H}-3,13-15$ & 27.89 & 34.2 & 8.4 & -0.62 & 1.18 & 44.5 & $925 \mathrm{D}-3 \mathrm{H}-5,123-125$ & 31.99 & 50.8 & 17.9 \\
\hline $925 \mathrm{D}-3 \mathrm{H}-3,18-20$ & 27.94 & 34.0 & 7.8 & -0.91 & 0.81 & & & 31.99 & & \\
\hline $925 \mathrm{D}-3 \mathrm{H}-3,23-25$ & 27.99 & 34.5 & 8.4 & -1.05 & 0.99 & 38.3 & 925D-3H-5, 128-130 & 32.04 & 43.1 & 14.4 \\
\hline & 27.99 & & & -0.88 & 1.05 & & & 32.04 & & \\
\hline 925D-3H-3, 28-30 & 28.04 & 31.3 & 7.8 & -1.02 & 1.02 & & 925D-3H-5, 133-135 & 32.09 & 45.9 & 15.3 \\
\hline $925 \mathrm{D}-3 \mathrm{H}-3,33-35$ & 28.09 & 30.4 & 8.2 & -1.01 & 1.06 & 41.7 & 925D-3H-5, 138-140 & 32.14 & 48.3 & 15.9 \\
\hline $925 \mathrm{D}-3 \mathrm{H}-3,38-40$ & 28.14 & 30.1 & 9.3 & -1.18 & 1.41 & & $925 \mathrm{D}-3 \mathrm{H}-5,143-145$ & 32.19 & 49.7 & 17.6 \\
\hline $925 \mathrm{D}-3 \mathrm{H}-3,43-45$ & 28.19 & 32.9 & 8.1 & -1.37 & 1.10 & 36.0 & $925 \mathrm{D}-3 \mathrm{H}-5,148-150$ & 32.24 & 45.1 & 16.1 \\
\hline $925 \mathrm{D}-3 \mathrm{H}-3,48-50$ & 28.24 & 30.7 & 8.9 & -1.13 & 1.22 & & $925 \mathrm{D}-3 \mathrm{H}-6,3-5$ & 32.29 & 33.1 & 11.9 \\
\hline $925 \mathrm{D}-3 \mathrm{H}-3,53-55$ & 28.29 & 25.2 & 4.2 & -1.07 & 1.53 & 44.3 & $925 \mathrm{C}-4 \mathrm{H}-3,82-83$ & 32.31 & 35.0 & 14.6 \\
\hline $925 \mathrm{D}-3 \mathrm{H}-3,58-60$ & 28.34 & 31.3 & 9.2 & -1.53 & 1.29 & & $925 \mathrm{D}-3 \mathrm{H}-6,8-10$ & 32.34 & 23.4 & 8.4 \\
\hline $925 \mathrm{D}-3 \mathrm{H}-3,63-65$ & 28.39 & 40.4 & 10.7 & -1.71 & 1.79 & 56.2 & $925 \mathrm{C}-4 \mathrm{H}-3,88-89$ & 32.37 & 29.2 & 6.3 \\
\hline $925 \mathrm{D}-3 \mathrm{H}-3,68-70$ & 28.44 & 26.7 & 9.0 & -1.22 & 0.98 & & $925 \mathrm{D}-3 \mathrm{H}-6,13-15$ & 32.39 & 29.0 & 9.1 \\
\hline $925 \mathrm{D}-3 \mathrm{H}-3,73-75$ & 28.49 & 27.6 & 7.2 & -1.04 & 0.76 & 65.4 & $925 \mathrm{C}-4 \mathrm{H}-3,93-94$ & 32.42 & 32.5 & 9.4 \\
\hline $925 \mathrm{D}-3 \mathrm{H}-3,78-80$ & 28.54 & 39.5 & 11.7 & -1.38 & 1.36 & & $925 \mathrm{D}-3 \mathrm{H}-6,18-20$ & 32.44 & 32.1 & 7.4 \\
\hline $925 \mathrm{D}-3 \mathrm{H}-3,83-85$ & 28.59 & 43.5 & 13.8 & -1.23 & 1.41 & 59.2 & $925 \mathrm{C}-4 \mathrm{H}-3,98-99$ & 32.47 & 31. & 7.5 \\
\hline $925 \mathrm{D}-3 \mathrm{H}-3,88-90$ & 28.64 & 46.3 & 14.5 & -1.44 & 1.18 & & $925 \mathrm{C}-4 \mathrm{H}-3,103-104$ & 32.52 & 35. & 7.6 \\
\hline $925 \mathrm{D}-3 \mathrm{H}-3,93-95$ & 28.69 & 50.3 & 18.6 & -1.46 & 1.05 & 29.8 & $925 \mathrm{C}-4 \mathrm{H}-3,108-109$ & 32.57 & 32 & 8.2 \\
\hline $925 \mathrm{D}-3 \mathrm{H}-3,98-100$ & 28.74 & 58.5 & 25.8 & -1.83 & 1.35 & & $925 \mathrm{C}-4 \mathrm{H}-3,113-114$ & 32.62 & 36.9 & 8.0 \\
\hline $925 \mathrm{D}-3 \mathrm{H}-3,103-105$ & 28.79 & 67.7 & 23.1 & -1.73 & 1.12 & 37.5 & $925 \mathrm{C}-4 \mathrm{H}-3,118-119$ & 32.67 & 34.0 & 8.6 \\
\hline $925 \mathrm{D}-3 \mathrm{H}-3,108-110$ & 28.84 & 58.8 & 17.4 & -1.71 & 1.21 & & $925 \mathrm{C}-4 \mathrm{H}-3,123-124$ & 32.72 & 28.7 & 5.8 \\
\hline-115 & 28.89 & 53.2 & 14.4 & -1.54 & 1.26 & 44.7 & $925 \mathrm{C}-4 \mathrm{H}-3,128-129$ & 32 & 18 & 3.3 \\
\hline $925 \mathrm{D}-3 \mathrm{H}-3,118-120$ & 28.94 & 55. & 17.6 & -1.13 & 0.98 & & $925 \mathrm{C}-4 \mathrm{H}-3,133-134$ & 32.82 & 26.4 & 6.9 \\
\hline $925 \mathrm{D}-3 \mathrm{H}-3,123-125$ & 28.99 & 30 & 9.5 & -1.27 & 1.03 & 34.6 & $925 \mathrm{C}-4 \mathrm{H}-3,138-139$ & 32.87 & 25.8 & 6.8 \\
\hline $925 \mathrm{D}-3 \mathrm{H}-3,128-130$ & 29.04 & 24 & 5.6 & -0.70 & 0.30 & & & 32.87 & & \\
\hline $925 \mathrm{D}-3 \mathrm{H}-3,133-135$ & 29.09 & 25.0 & 6.0 & -0.42 & 0.68 & 42.1 & $925 \mathrm{C}-4 \mathrm{H}-3,143-144$ & 32.92 & 21 & 3.8 \\
\hline $3,138-140$ & 29.14 & 23.0 & 4.6 & -0.54 & 0.73 & & $925 \mathrm{C}-4 \mathrm{H}-3,148-149$ & 32.97 & 27 & 6.7 \\
\hline $3,143-145$ & 29.19 & 23.7 & 4.3 & -0.78 & 0.71 & 43.6 & & 33. & 30 & 5.3 \\
\hline$-3 \mathrm{H}-3,148-150$ & 29 & 23 & 5. & -0 & 1.12 & & 92 & 33 & 43 & 10.0 \\
\hline $925 \mathrm{D}-3 \mathrm{H}-4,3-5$ & 29.29 & 23.7 & 4.8 & -0.82 & 0.74 & 33.0 & 1 & 33 & 42 & 12.5 \\
\hline $925 \mathrm{D}-3 \mathrm{H}-4,8-10$ & 29.34 & 26.1 & 6.9 & -0.77 & 0.96 & & $925 \mathrm{C}-4 \mathrm{H}-4,18-19$ & 33.17 & 36 & 9.0 \\
\hline $925 \mathrm{D}-3 \mathrm{H}-4,13-15$ & 29.39 & 28.5 & 6.0 & -0.92 & 0.95 & 64.2 & $925 \mathrm{C}-4 \mathrm{H}-4,23-24$ & 33.22 & 38.9 & 14.8 \\
\hline$-4,18-20$ & 29.44 & 29 & 7.3 & -0.84 & 1.05 & 04.2 & $925 \mathrm{C}-4 \mathrm{H}-4,28-29$ & 33.27 & 49.8 & 12.8 \\
\hline $925 \mathrm{D}-3 \mathrm{H}-4,23-25$ & 29. & 37 & 8.4 & -0 . & 1.51 & 43.3 & $925 \mathrm{C}-4 \mathrm{H}-4,33-34$ & 33. & 52 & 7 \\
\hline$-3 \mathrm{H}-4,28-30$ & & & 10.3 & -1 & 0. & T. & & 3. & 5 & 19 \\
\hline $925 \mathrm{D}-3 \mathrm{H}-4,33-35$ & 29. & 37 & 8.8 & -1.28 & 1.15 & 32.3 & 92 & 33.42 & 51 & 21.4 \\
\hline $925 \mathrm{D}-3 \mathrm{H}-4,38-40$ & 29.64 & 33.8 & 9.0 & -1.21 & 0.91 & & $925 \mathrm{C}-4 \mathrm{H}-4,48-49$ & 33.47 & 68.8 & 20.6 \\
\hline $925 \mathrm{D}-3 \mathrm{H}-4,43-45$ & 29.69 & 36.2 & 10.4 & -1.51 & 1.29 & 39.4 & $925 \mathrm{C}-4 \mathrm{H}-4,53-54$ & 33.52 & 67.2 & 22.4 \\
\hline $48-50$ & 29.74 & 40 & 10.1 & -1.37 & 1.51 & & & 33.52 & & \\
\hline 53-55 & 29.79 & 36 & 12.0 & -1 . & 0.90 & 57 & $925 \mathrm{C}-$ & 335 & 66 & 21 \\
\hline$-3 \mathrm{H}-4,58-60$ & & 43 & 10 & -1 & 0.92 & & & & & \\
\hline $925 \mathrm{D}-3 \mathrm{H}-4,63-65$ & 29.89 & 41.0 & 12.2 & -1.59 & 1.14 & 46.5 & $925 \mathrm{C}-4 \mathrm{H}-4,68$ & 33.67 & 59 & 16.1 \\
\hline $925 \mathrm{D}-3 \mathrm{H}-4,68-70$ & 29.94 & 37.8 & 14.4 & -1.41 & 1.20 & & $925 \mathrm{C}-4 \mathrm{H}-4,73-74$ & 33.72 & 44.3 & 8.2 \\
\hline $925 \mathrm{D}-3 \mathrm{H}-4,73-75$ & 29.99 & 34.6 & 24.6 & -1.49 & 1.17 & 50.0 & $925 \mathrm{C}-4 \mathrm{H}-4,78-79$ & 33.77 & 37.6 & 6.7 \\
\hline $925 \mathrm{D}-3 \mathrm{H}-4,78-80$ & 30.04 & 39.2 & 10.4 & & 12 & 32.0 & $925 \mathrm{C}-4 \mathrm{H}-4,83-84$ & 3382 & & 4.4 \\
\hline & 30.04 & & & -1.17 & 1.45 & & $925 \mathrm{C}-4 \mathrm{H}-4,88-89$ & 33.87 & 36.6 & 11.0 \\
\hline
\end{tabular}


Table 1 (continued).

\begin{tabular}{|c|c|c|c|c|c|c|}
\hline $\begin{array}{l}\text { Hole, core, section } \\
\text { interval }(\mathrm{cm})\end{array}$ & $\begin{array}{l}\text { Depth } \\
\text { (mcd) }\end{array}$ & $\begin{array}{c}\mathrm{CaCO}_{3} \\
(\%)\end{array}$ & $\begin{array}{c}>63 \mu \mathrm{m} \\
(\%)\end{array}$ & $\begin{array}{c}\delta^{18} \mathrm{O} \\
(\%)\end{array}$ & $\begin{array}{c}\delta^{13} \mathrm{C} \\
(\%)\end{array}$ & $\begin{array}{c}F . \\
\text { profunda } \\
(\%)\end{array}$ \\
\hline $925 \mathrm{C}-4 \mathrm{H}-4,93-94$ & 33.92 & 51.6 & 14.5 & -1.04 & 1.43 & 17.7 \\
\hline 925C-4H-4, 98-99 & 33.97 & 47.8 & 10.2 & -1.18 & 0.97 & 12.8 \\
\hline $925 \mathrm{C}-4 \mathrm{H}-4,103-104$ & 34.02 & 50.0 & 10.3 & -1.23 & 1.48 & 14.6 \\
\hline $925 \mathrm{C}-4 \mathrm{H}-4,108-109$ & 34.07 & 43.6 & 11.8 & -1.41 & 1.38 & 9.6 \\
\hline $925 \mathrm{C}-4 \mathrm{H}-4,113-114$ & 34.12 & 44.7 & 13.0 & -1.35 & 1.18 & 8.0 \\
\hline $925 \mathrm{C}-4 \mathrm{H}-4,118-119$ & 34.17 & 49.4 & 14.4 & -1.46 & 1.60 & 25.9 \\
\hline $925 \mathrm{C}-4 \mathrm{H}-4,123-124$ & 34.22 & 55.4 & 14.0 & -1.59 & 1.69 & 27.9 \\
\hline $925 \mathrm{C}-4 \mathrm{H}-4,128-129$ & $\begin{array}{l}34.27 \\
34.27\end{array}$ & 50.6 & 16.3 & $\begin{array}{l}-1.37 \\
-1.35\end{array}$ & $\begin{array}{l}1.49 \\
1.49\end{array}$ & 52.1 \\
\hline $925 \mathrm{C}-4 \mathrm{H}-4,133-134$ & 34.32 & 63.7 & 18.8 & -1.64 & 1.11 & 32.4 \\
\hline $925 \mathrm{C}-4 \mathrm{H}-4,138-139$ & 34.37 & 52.8 & 18.6 & -1.54 & 1.15 & 27.5 \\
\hline $925 \mathrm{C}-4 \mathrm{H}-4,143-144$ & 34.42 & 52.9 & 13.6 & -1.47 & 0.90 & 38.0 \\
\hline $925 \mathrm{C}-4 \mathrm{H}-4,148-149$ & 34.47 & 27.4 & 7.9 & -1.20 & 1.62 & 42.7 \\
\hline $925 \mathrm{C}-4 \mathrm{H}-5,3-4$ & 34.52 & 31.5 & 4.3 & -1.13 & 1.19 & 40.8 \\
\hline $925 \mathrm{C}-4 \mathrm{H}-5,8-9$ & 34.57 & 38.8 & 6.4 & -1.26 & 1.37 & 22.5 \\
\hline $925 \mathrm{C}-4 \mathrm{H}-5,13-14$ & 34.62 & 42.0 & 11.3 & $\begin{array}{l}-1.20 \\
-1.22\end{array}$ & 1.24 & 30.0 \\
\hline $925 \mathrm{C}-4 \mathrm{H}-5,18-19$ & 34.67 & 34.6 & 5.9 & -1.32 & 1.24 & 33.8 \\
\hline $925 \mathrm{C}-4 \mathrm{H}-5,23-24$ & 34.72 & 49.0 & 8.3 & -1.32 & 0.98 & 30.7 \\
\hline $925 \mathrm{C}-4 \mathrm{H}-5,28-29$ & 34.77 & 48.0 & 11.5 & -1.36 & 1.25 & 44.5 \\
\hline $925 \mathrm{C}-4 \mathrm{H}-5,33-34$ & 34.82 & 60.5 & 13.8 & -1.70 & 1.85 & 30.9 \\
\hline $925 \mathrm{C}-4 \mathrm{H}-5,38-39$ & 34.87 & 63.7 & 15.7 & -1.65 & 1.56 & 40.0 \\
\hline $925 \mathrm{C}-4 \mathrm{H}-5,43-44$ & 34.92 & 61.2 & 16.8 & $\begin{array}{l}-1.05 \\
-1.64\end{array}$ & 1.22 & 32.5 \\
\hline $925 \mathrm{C}-4 \mathrm{H}-5,48-49$ & 34.97 & 58.7 & 14.5 & -1.53 & 1.49 & 27.8 \\
\hline $925 \mathrm{C}-4 \mathrm{H}-5,53-54$ & 35.02 & 57.4 & 13.6 & -1.30 & 1.22 & 27.7 \\
\hline $925 \mathrm{C}-4 \mathrm{H}-5,58-59$ & 35.07 & 49.7 & 14.0 & -1.42 & 1.17 & 20.9 \\
\hline $925 \mathrm{C}-4 \mathrm{H}-5,63-64$ & 35.12 & 42.7 & 10.5 & -1.11 & 1.30 & 15.0 \\
\hline $925 \mathrm{C}-4 \mathrm{H}-5,68-69$ & 35.17 & 39.4 & 12.1 & -1.12 & 1.28 & 33.5 \\
\hline $925 \mathrm{C}-4 \mathrm{H}-5,73-74$ & 35.22 & 45.5 & 12.9 & -1.01 & $\begin{array}{l}1.20 \\
2.04\end{array}$ & 24.2 \\
\hline $925 \mathrm{C}-4 \mathrm{H}-5,78-79$ & 35.27 & 29.6 & 7.3 & -1.17 & 1.15 & 31.1 \\
\hline $925 \mathrm{C}-4 \mathrm{H}-5,83-84$ & 35.32 & 41.0 & 6.9 & -1.33 & 1.53 & 41.2 \\
\hline $925 \mathrm{C}-4 \mathrm{H}-5,88-89$ & 35.37 & 33.2 & 4.5 & -1.38 & 1.63 & 53.7 \\
\hline $925 \mathrm{C}-4 \mathrm{H}-5,93-94$ & 35.42 & 43.9 & 10.7 & -1.47 & 1.60 & 49.1 \\
\hline $925 \mathrm{C}-4 \mathrm{H}-5,98-99$ & 35.47 & 42.5 & 14.9 & -1.52 & 1.39 & 34.3 \\
\hline 925C-4H-5, 103-104 & 35.52 & 40.3 & 13.8 & $\begin{array}{l}-1.52 \\
-1.32\end{array}$ & 1.46 & 34.2 \\
\hline $925 \mathrm{C}-4 \mathrm{H}-5,108-109$ & 35.57 & 31.3 & 7.0 & -1.23 & 1.54 & 23.8 \\
\hline $925 \mathrm{C}-4 \mathrm{H}-5,113-114$ & 35.62 & 48.1 & 12.2 & -1.41 & 1.52 & 44.8 \\
\hline $925 \mathrm{C}-4 \mathrm{H}-5,118-119$ & 35.67 & 58.9 & 20.2 & -1.49 & 1.49 & 58.1 \\
\hline $925 \mathrm{C}-4 \mathrm{H}-5,123-124$ & 35.72 & 72.3 & 25.3 & -1.32 & 1.43 & 56.7 \\
\hline $925 \mathrm{C}-4 \mathrm{H}-5,128-129$ & 35.77 & 59.2 & 22.8 & $\begin{array}{l}-1.39 \\
-1.39\end{array}$ & 1.30 & 35.9 \\
\hline $925 \mathrm{C}-4 \mathrm{H}-5,133-134$ & 35.82 & 56.5 & 12.8 & $\begin{array}{l}-1.59 \\
-1.25\end{array}$ & 0.86 & 35.5 \\
\hline $925 \mathrm{C}-4 \mathrm{H}-5,138-139$ & 35.87 & 48.0 & 13.3 & -1.10 & 0.70 & 30.3 \\
\hline $925 \mathrm{C}-4 \mathrm{H}-5,143-144$ & 35.92 & 56.8 & 14.5 & -1.27 & 1.20 & 33.9 \\
\hline $925 \mathrm{C}-4 \mathrm{H}-5,148-149$ & 35.97 & 54.8 & 14.6 & -1.17 & 1.18 & 13.1 \\
\hline $925 \mathrm{C}-4 \mathrm{H}-6,3-4$ & 36.02 & 53.9 & 13.2 & -1.29 & 0.32 & 25.0 \\
\hline $925 \mathrm{C}-4 \mathrm{H}-6,8-9$ & 36.07 & 53.6 & 14.8 & -1.43 & 0.78 & 23.3 \\
\hline $925 \mathrm{C}-4 \mathrm{H}-6,13-14$ & 36.12 & 45.3 & 12.5 & -1.39 & 0.45 & 16.4 \\
\hline $925 \mathrm{C}-4 \mathrm{H}-6,18-19$ & 36.17 & 45.0 & 11.8 & -1.38 & 1.20 & 18.4 \\
\hline $925 \mathrm{C}-4 \mathrm{H}-6,23-24$ & 36.22 & 42.9 & 11.0 & -1.39 & 0.70 & $\begin{array}{l}10.4 \\
33.7\end{array}$ \\
\hline $925 \mathrm{C}-4 \mathrm{H}-6,28-29$ & 36.27 & 38.5 & 10.2 & -1.17 & 0.78 & 51.1 \\
\hline $925 \mathrm{C}-4 \mathrm{H}-6,33-34$ & 36.32 & 35.5 & 10.6 & -1.20 & 0.70 & 67.0 \\
\hline $925 \mathrm{C}-4 \mathrm{H}-6,38-39$ & 36.37 & 34.6 & 11.7 & -1.28 & 0.26 & 61.6 \\
\hline $925 \mathrm{C}-4 \mathrm{H}-6,43-44$ & 36.42 & 30.3 & 8.7 & -1.15 & 0.96 & $\begin{array}{l}0.0 \\
54.4\end{array}$ \\
\hline $925 \mathrm{D}-4 \mathrm{H}-2,20-21$ & 36.47 & & 9.5 & -1.12 & 1.19 & 66.2 \\
\hline $925 \mathrm{C}-4 \mathrm{H}-6,53-54$ & 36.52 & 26.5 & 8.7 & $\begin{array}{l}-1.12 \\
-1.24\end{array}$ & 1.18 & 64.1 \\
\hline $925 \mathrm{D}-4 \mathrm{H}-2,25-26$ & 36.52 & & 12.9 & -1.25 & 0.93 & \\
\hline $925 \mathrm{C}-4 \mathrm{H}-6,58-59$ & 36.57 & 36.8 & 11.7 & -1.33 & 1.00 & 49.2 \\
\hline $925 \mathrm{D}-4 \mathrm{H}-2,30-31$ & 36.57 & 31.7 & 14.8 & -1.23 & 1.28 & 44.8 \\
\hline $925 \mathrm{D}-4 \mathrm{H}-2,35-36$ & 36.62 & & $\begin{array}{l}11.0 \\
21.3\end{array}$ & $\begin{array}{l}-1.23 \\
-1.59\end{array}$ & 1.27 & \\
\hline $925 \mathrm{D}-4 \mathrm{H}-2,40-41$ & 36.67 & 62.1 & 25.7 & -1.90 & 1.45 & 47.0 \\
\hline $925 \mathrm{D}-4 \mathrm{H}-2,45-46$ & 36.72 & & 30.5 & -1.97 & 1.34 & \\
\hline $925 \mathrm{D}-4 \mathrm{H}-2,50-51$ & 36.77 & 63.6 & 32.7 & -1.97 & 1.35 & 37.1 \\
\hline $925 \mathrm{D}-4 \mathrm{H}-2,55-56$ & 36.82 & & 31.0 & -1.86 & 1.64 & \\
\hline $925 \mathrm{D}-4 \mathrm{H}-2,60-61$ & 36.87 & & 29.4 & $\begin{array}{l}-1.00 \\
-1.67\end{array}$ & 1.43 & 54.3 \\
\hline $925 \mathrm{D}-4 \mathrm{H}-2,65-66$ & 36.92 & & 33.6 & $\begin{array}{l}-1.01 \\
-1.70\end{array}$ & 1.45 & 34.5 \\
\hline $925 \mathrm{D}-4 \mathrm{H}-2,70-71$ & 36.97 & 61.5 & 27.3 & -1.76 & 1.34 & 68.6 \\
\hline $925 \mathrm{D}-4 \mathrm{H}-2,75-76$ & 37.02 & & 25.4 & $\begin{array}{l}-1.69 \\
-1.69\end{array}$ & 1.58 & \\
\hline $925 \mathrm{D}-4 \mathrm{H}-2,80-81$ & 37.07 & 53.3 & 23.3 & -1.42 & 1.35 & 24.5 \\
\hline $925 \mathrm{D}-4 \mathrm{H}-2,85-86$ & 37.12 & & 21.5 & -1.43 & 1.17 & \\
\hline $925 \mathrm{D}-4 \mathrm{H}-2,90-91$ & 37.17 & 48.8 & 20.7 & -1.53 & 1.44 & 29.5 \\
\hline $925 \mathrm{D}-4 \mathrm{H}-2,95-96$ & 37.22 & & 20.7 & $\begin{array}{l}-1.53 \\
-1.50\end{array}$ & $\begin{array}{l}1.44 \\
0.99\end{array}$ & 29.3 \\
\hline $925 \mathrm{D}-4 \mathrm{H}-2,100-101$ & 37.27 & 46.7 & 12.3 & -1.54 & 1.06 & 29.7 \\
\hline $925 \mathrm{D}-4 \mathrm{H}-2,105-106$ & 37.32 & & 15.1 & -1.45 & 1.04 & \\
\hline $925 \mathrm{D}-4 \mathrm{H}-2,110-111$ & 37.37 & & 16.0 & -1.44 & 0.93 & 38.3 \\
\hline $925 \mathrm{D}-4 \mathrm{H}-2,115-116$ & 37.42 & & 17.7 & -1.30 & 1.03 & \\
\hline $925 \mathrm{D}-4 \mathrm{H}-2,120-121$ & 37.47 & 46.2 & 16.5 & -1.20 & 1.13 & 34.2 \\
\hline $925 \mathrm{D}-4 \mathrm{H}-2,125-126$ & 37.52 & & 11.8 & $\begin{array}{l}-1.20 \\
-1.15\end{array}$ & 0.77 & \\
\hline $925 \mathrm{D}-4 \mathrm{H}-2,130-131$ & 37.5 & 27.5 & 8.1 & -0.90 & 1.41 & 50.0 \\
\hline $925 \mathrm{D}-4 \mathrm{H}-2,135-136$ & 37.62 & & 5.2 & -0.87 & 1.17 & \\
\hline $925 \mathrm{D}-4 \mathrm{H}-2,140-141$ & 37.67 & 22.8 & 6.6 & -1.12 & 0.96 & 80.5 \\
\hline $925 \mathrm{D}-4 \mathrm{H}-2,145-146$ & 37.72 & 26.6 & 7.6 & -1.20 & 1.18 & \\
\hline $925 \mathrm{D}-4 \mathrm{H}-3,20-21$ & 37.97 & & 20.2 & -1.48 & 1.22 & \\
\hline
\end{tabular}

Spectral Analysis

Spectral analyses were performed on $\delta^{18} \mathrm{O}$, carbonate MAR, coarse-fraction content, and Florisphaera profunda percentage records from Site 925 (Fig. 9). Data were interpolated to a constant time interval of 1 k.y. and linearly detrended. Power spectra calculated according to the Blackman-Tukey method with a Hanning window and a $30 \%$ lag (138 points) resulted in a bandwidth of about 0.012 . The $\delta^{18} \mathrm{O}$ record is the only one that shows spectral variance occurring at a frequency approaching $\sim 100 \mathrm{k} . \mathrm{y} .^{-1}$ (Fig. 9). The other power spectra show important amounts of variance at lower frequencies. However, these spectral peaks should be regarded with great caution as the short length of the interval studied (covering about 420 k.y.) and the likely change in periodic behavior do not allow us to address those frequencies. These low-frequency peaks in the power spectra most probably correspond to noncyclic, long-term variations that are "misinterpreted" by the spectral analysis calculation. With the exception of these low-frequency peaks, we conclude that there is no evidence during the mid-Pleistocene transition interval of climatic responses other than those occurring at the primary Milankovitch frequency bands (namely, obliquity, at $1 / 41 \mathrm{k} . \mathrm{y} .^{-1}$, and precession, at $1 /$ 23 and $\left.1 / 19 \mathrm{k} . \mathrm{y} \cdot{ }^{-1}\right)$. This confirms the conclusion drawn by Ruddiman et al. (1989) from their detailed study of various climatic indicators from DSDP Site 607 in the North Atlantic. In the dust flux records from ODP Site 659 in the eastern equatorial Atlantic, however, the mid-Pleistocene transition interval is characterized by the almost complete disappearance of the precession-related oscillations and the development of strong oscillations at non-Milankovitch primary frequencies (see fig. 9 in Tiedemann et al., 1994). It is not clear, however, whether this dust flux behavior represents a true signal (and may reflect, therefore, possible transient, nonlinear responses of the climatic system to insolation forcing) or if these non-Milankovitch frequencies arise from some problems in the age model or in the dust record.

All the power spectra records from Site 925 show the clear dominance of obliquity-related 41-k.y. oscillations and the relative weak development of precession-related oscillations (23 and 19 k.y.). In the carbonate MAR spectra, the variance associated with the 41-k.y. oscillation represents about $40 \%$ of the total variance whereas the precession-related oscillations $(23+19$ k.y. $)$ only account for about $20 \%$ of the total variance. In the record of $F$. profunda, $29 \%$ of the total variance is accounted for by obliquity-related oscillations and only $12 \%$ by the precession-related oscillations (the same values were obtained for the $\delta^{18} \mathrm{O}$ record).

It is well known from insolation calculations and paleoclimatic evidence that obliquity-driven changes have a dominant effect on seasonal and mean annual solar radiation budgets at high latitudes but have small effect on changes on low-latitude insolation (Berger and Loutre, 1994). Our spectral analysis results suggest therefore that biogenic sedimentation and thermocline dynamics on Ceara Rise in the time interval 680-1100 ka were responding mostly to high-latitude climatic processes.

\section{Filtering}

Visual examination of the time series shown in Figures 5 and 6 suggests a complex evolution of phase relationships among $\delta^{18} \mathrm{O}$, carbonate MAR, and $F$. profunda abundance records at the Milankovitch frequencies. Cross-spectral analysis estimates phase relationships between two time series over the entire frequency range they cover, but calculates only mean phase and does not address possible transient changes in leads and lags. Thus, to address in detail the possible evolution of leads and lags through the 680- to 1100-ka time interval, and especially across glacial isotopic Stage 22, we decided to compare in the time domain the Milankovitch-related oscillations obtained by filtering the $\delta^{18} \mathrm{O}$, carbonate MAR, and $F$. profunda time se- 
Table 2. Carbonate content and sand-sized fraction content measured from Site 928 samples.

\begin{tabular}{|c|c|c|c|c|c|c|}
\hline $\begin{array}{l}\text { Hole, core, section, } \\
\text { interval }(\mathrm{cm})\end{array}$ & $\begin{array}{l}\text { Depth } \\
\text { (mcd) }\end{array}$ & $\begin{array}{c}\mathrm{CaCO}_{3} \\
(\%)\end{array}$ & $\begin{array}{c}>63 \mu \mathrm{m} \\
(\%)\end{array}$ & $\begin{array}{l}\text { Hole, core, section, } \\
\quad \text { interval }(\mathrm{cm})\end{array}$ & $\begin{array}{l}\text { Depth } \\
\text { (mcd) }\end{array}$ & $\begin{array}{c}\mathrm{CaCO}_{3} \\
(\%)\end{array}$ \\
\hline $928 \mathrm{~A}-3 \mathrm{H}-3,10-12$ & 23.24 & & 13.8 & $928 \mathrm{~A}-3 \mathrm{H}-6,100-101$ & 28.64 & \\
\hline $928 \mathrm{~A}-3 \mathrm{H}-3,20-22$ & 23.34 & & 31.3 & $928 \mathrm{~A}-3 \mathrm{H}-6,105-106$ & 28.69 & \\
\hline $928 \mathrm{~A}-3 \mathrm{H}-3,30-32$ & 23.44 & & 24.3 & $928 \mathrm{~A}-3 \mathrm{H}-6,110-111$ & 28.74 & 27.9 \\
\hline $928 \mathrm{~A}-3 \mathrm{H}-3,40-42$ & 23.54 & & 9.0 & $928 \mathrm{~A}-3 \mathrm{H}-6,115-116$ & 28.79 & \\
\hline $928 \mathrm{~A}-3 \mathrm{H}-3,50-52$ & 23.64 & & 2.5 & $928 \mathrm{~A}-3 \mathrm{H}-6,120-121$ & 28.84 & 31.2 \\
\hline $928 \mathrm{~A}-3 \mathrm{H}-3,60-62$ & 23.74 & & 3.6 & $928 \mathrm{~A}-3 \mathrm{H}-6,125-126$ & 28.89 & 30.7 \\
\hline $928 \mathrm{~A}-3 \mathrm{H}-3,70-72$ & 23.84 & & 5.6 & $928 \mathrm{~B}-4 \mathrm{H}-2,2-3$ & 28.92 & 35.2 \\
\hline $928 \mathrm{~A}-3 \mathrm{H}-3,80-82$ & 23.94 & & 3.9 & $928 \mathrm{~A}-3 \mathrm{H}-6,130-131$ & 28.94 & 40.9 \\
\hline $928 \mathrm{~A}-3 \mathrm{H}-3,96-97$ & 24.10 & & 4.4 & $928 \mathrm{~B}-4 \mathrm{H}-2,7-8$ & 28.97 & 47.5 \\
\hline $928 \mathrm{~A}-3 \mathrm{H}-3,105-106$ & 24.19 & & 7.6 & $928 \mathrm{~A}-3 \mathrm{H}-6,135-136$ & 28.99 & 47.2 \\
\hline $928 \mathrm{~A}-3 \mathrm{H}-3,110-111$ & 24.24 & & 2.2 & $928 \mathrm{~B}-4 \mathrm{H}-2,12-13$ & 29.02 & 49.7 \\
\hline $928 \mathrm{~A}-3 \mathrm{H}-3,115-116$ & 24.29 & & 2.1 & $928 \mathrm{~A}-3 \mathrm{H}-6,140-141$ & 29.04 & 49.7 \\
\hline $928 \mathrm{~A}-3 \mathrm{H}-3,125-126$ & 24.39 & & 6.8 & $928 \mathrm{~B}-4 \mathrm{H}-2,17-18$ & 29.07 & 41.6 \\
\hline $928 \mathrm{~A}-3 \mathrm{H}-3,135-136$ & 24.49 & & 4.5 & $928 \mathrm{~A}-3 \mathrm{H}-6,145-146$ & 29.09 & 55.1 \\
\hline $928 \mathrm{~A}-3 \mathrm{H}-4,0-2$ & 24.64 & & 3.2 & $928 \mathrm{~B}-4 \mathrm{H}-2,22-23$ & 29.12 & 48.9 \\
\hline $928 \mathrm{~A}-3 \mathrm{H}-4,10-12$ & 24.74 & & 1.9 & $928 \mathrm{~B}-4 \mathrm{H}-2,27-28$ & 29.17 & 38.4 \\
\hline $928 \mathrm{~A}-3 \mathrm{H}-4,20-22$ & 24.84 & & 3.4 & $928 \mathrm{~B}-4 \mathrm{H}-2,32-33$ & 29.22 & 26.0 \\
\hline $928 \mathrm{~A}-3 \mathrm{H}-4,30-32$ & 24.94 & & 0.6 & $928 \mathrm{~B}-4 \mathrm{H}-2,37-38$ & 29.27 & 17.7 \\
\hline $928 \mathrm{~A}-3 \mathrm{H}-4,35-37$ & 24.99 & 18.5 & 1.5 & $928 \mathrm{~B}-4 \mathrm{H}-2,42-43$ & 29.32 & 5.3 \\
\hline $928 \mathrm{~A}-3 \mathrm{H}-4,40-42$ & 25.04 & 26.5 & 2.6 & $928 \mathrm{~B}-4 \mathrm{H}-2,47-48$ & 29.37 & 11.6 \\
\hline $928 \mathrm{~A}-3 \mathrm{H}-4,45-47$ & 25.09 & 23.1 & 2.3 & $928 \mathrm{~B}-4 \mathrm{H}-2,52-53$ & 29.42 & 8.4 \\
\hline $928 \mathrm{~A}-3 \mathrm{H}-4,50-52$ & 25.14 & 14.8 & 0.9 & $928 \mathrm{~B}-4 \mathrm{H}-2,57-58$ & 29.47 & 10.6 \\
\hline $928 \mathrm{~A}-3 \mathrm{H}-4,55-57$ & 25.19 & 4.6 & 0.3 & $928 \mathrm{~B}-4 \mathrm{H}-2,62-63$ & 29.52 & 9.0 \\
\hline $928 \mathrm{~A}-3 \mathrm{H}-4,60-62$ & 25.24 & 6.9 & 0.2 & $928 \mathrm{~B}-4 \mathrm{H}-2,67-68$ & 29.57 & 9.8 \\
\hline $928 \mathrm{~A}-3 \mathrm{H}-4,65-67$ & 25.29 & 12.8 & 0.8 & $928 \mathrm{~B}-4 \mathrm{H}-2,72-73$ & 29.62 & $\begin{array}{r}31.0 \\
11.4\end{array}$ \\
\hline $928 \mathrm{~A}-3 \mathrm{H}-4,70-72$ & 25.34 & 9.4 & 0.4 & $928 \mathrm{~B}-4 \mathrm{H}-2,77-78$ & 29.67 & 9.5 \\
\hline $928 \mathrm{~A}-3 \mathrm{H}-4,75-77$ & 25.39 & 13.7 & 1.1 & $928 \mathrm{~B}-4 \mathrm{H}-2,82-83$ & 29.72 & 12.7 \\
\hline $928 \mathrm{~A}-3 \mathrm{H}-4,80-82$ & 25.44 & 19.4 & 1.6 & $928 \mathrm{~B}-4 \mathrm{H}-2,87-88$ & 29.77 & 20.9 \\
\hline $928 \mathrm{~A}-3 \mathrm{H}-4,85-87$ & 25.49 & & 2.2 & $928 \mathrm{~B}-4 \mathrm{H}-2,92-93$ & 29.82 & 17.5 \\
\hline $928 \mathrm{~A}-3 \mathrm{H}-4,90-91$ & 25.54 & & 2.8 & $928 \mathrm{~B}-4 \mathrm{H}-2,97-98$ & 29.87 & 20.5 \\
\hline $928 \mathrm{~A}-3 \mathrm{H}-4,95-96$ & 25.59 & 22.5 & 1.6 & $928 \mathrm{~B}-4 \mathrm{H}-2,102-103$ & 29.92 & 19.6 \\
\hline $928 \mathrm{~A}-3 \mathrm{H}-4,100-101$ & 25.64 & & 0.7 & $928 \mathrm{~B}-4 \mathrm{H}-2,107-108$ & 29.97 & 27.5 \\
\hline $928 \mathrm{~A}-3 \mathrm{H}-4,105-106$ & 25.69 & 13.7 & 1.1 & $928 \mathrm{~B}-4 \mathrm{H}-2,112-113$ & 30.02 & 29.4 \\
\hline $928 \mathrm{~A}-3 \mathrm{H}-4,110-111$ & 25.74 & & 1.1 & $928 \mathrm{~B}-4 \mathrm{H}-2,117-118$ & 30.07 & 29.6 \\
\hline $928 \mathrm{~A}-3 \mathrm{H}-4,115-116$ & 25.79 & 14.2 & 1.5 & $928 \mathrm{~B}-4 \mathrm{H}-2,122-123$ & 30.12 & 29.9 \\
\hline $928 \mathrm{~A}-3 \mathrm{H}-4,120-121$ & 25.84 & 37.9 & 1.0 & $928 \mathrm{~B}-4 \mathrm{H}-2,127-128$ & 30.17 & 29.9 \\
\hline $928 \mathrm{~A}-3 \mathrm{H}-4,125-126$ & 25.89 & 63.0 & 18.1 & $928 \mathrm{~B}-4 \mathrm{H}-2,132-133$ & 30.22 & 23.8 \\
\hline $928 \mathrm{~A}-3 \mathrm{H}-4,130-131$ & 25.94 & 60.4 & 20.2 & $928 \mathrm{~B}-4 \mathrm{H}-2,137-138$ & 30.27 & 21.2 \\
\hline $928 \mathrm{~A}-3 \mathrm{H}-4,135-136$ & 25.99 & 51.9 & 15.6 & $928 \mathrm{~B}-4 \mathrm{H}-2,142-143$ & 30.32 & 25.4 \\
\hline $928 \mathrm{~A}-3 \mathrm{H}-4,140-141$ & 26.04 & & 14.9 & $928 \mathrm{~B}-4 \mathrm{H}-2,147-148$ & 30.37 & 29.9 \\
\hline $928 \mathrm{~A}-3 \mathrm{H}-4,145-146$ & 26.09 & 45.6 & 16.7 & $928 \mathrm{~B}-4 \mathrm{H}-3,2-3$ & 30.42 & 25.7 \\
\hline $928 \mathrm{~A}-3 \mathrm{H}-5,0-2$ & 26.14 & 45.0 & 18.3 & $928 \mathrm{~B}-4 \mathrm{H}-3,7-8$ & 30.47 & 14.1 \\
\hline $928 \mathrm{~A}-3 \mathrm{H}-5,5-7$ & 26.19 & 41.0 & 11.7 & $928 \mathrm{~B}-4 \mathrm{H}-3,12-13$ & 30.52 & $\begin{array}{r}1.1 \\
8.0\end{array}$ \\
\hline $928 \mathrm{~A}-3 \mathrm{H}-5,10-12$ & 26.24 & 39.9 & 11.3 & $928 \mathrm{~B}-4 \mathrm{H}-3,17-18$ & 30.57 & 15.2 \\
\hline $928 \mathrm{~A}-3 \mathrm{H}-5,15-17$ & 26.29 & 38.4 & 13.0 & $928 \mathrm{~B}-4 \mathrm{H}-3,22-23$ & 30.62 & 28.5 \\
\hline $928 \mathrm{~A}-3 \mathrm{H}-5,20-22$ & 26.34 & 38.2 & 14.0 & $928 \mathrm{~B}-4 \mathrm{H}-3,27-28$ & 30.67 & 32.7 \\
\hline $928 \mathrm{~A}-3 \mathrm{H}-5,25-27$ & 26.39 & 37.9 & 16.1 & $928 \mathrm{~B}-4 \mathrm{H}-3,32-33$ & 30.72 & 38.0 \\
\hline $928 \mathrm{~A}-3 \mathrm{H}-5,30-32$ & 26.44 & 43.9 & 18.6 & $928 \mathrm{~B}-4 \mathrm{H}-3,37-38$ & 30.77 & 28.7 \\
\hline $928 \mathrm{~A}-3 \mathrm{H}-5,35-37$ & 26.49 & 46.7 & $\begin{array}{l}16.0 \\
16.1\end{array}$ & $928 \mathrm{~B}-4 \mathrm{H}-3,42-43$ & 30.82 & 19.1 \\
\hline $928 \mathrm{~A}-3 \mathrm{H}-5,40-42$ & 26.54 & 47.3 & 17.5 & $928 \mathrm{~B}-4 \mathrm{H}-3,47-48$ & 30.87 & 15.6 \\
\hline $928 \mathrm{~A}-3 \mathrm{H}-5,45-47$ & 26.59 & 42.2 & 7.9 & $928 \mathrm{~B}-4 \mathrm{H}-3,52-53$ & 30.92 & 30.4 \\
\hline $928 \mathrm{~A}-3 \mathrm{H}-5,50-52$ & 26.64 & 28.8 & 4.8 & $928 \mathrm{~B}-4 \mathrm{H}-3,57-58$ & 30.97 & 40.1 \\
\hline $928 \mathrm{~A}-3 \mathrm{H}-5,55-57$ & 26.69 & 17.2 & 1.6 & $928 \mathrm{~B}-4 \mathrm{H}-3,62-63$ & 31.02 & 47.8 \\
\hline $928 \mathrm{~A}-3 \mathrm{H}-5,60-62$ & 26.74 & 14.4 & 2.4 & $928 \mathrm{~B}-4 \mathrm{H}-3,67-68$ & 31.07 & 48.6 \\
\hline $928 \mathrm{~A}-3 \mathrm{H}-5,64-66$ & 26.78 & 15.3 & 2.5 & $928 \mathrm{~B}-4 \mathrm{H}-3,72-73$ & 31.12 & $\begin{array}{l}4.0 \\
39.0\end{array}$ \\
\hline $928 \mathrm{~A}-3 \mathrm{H}-5,70-72$ & 26.84 & 13.3 & 1.7 & $928 \mathrm{~B}-4 \mathrm{H}-3,77-78$ & 31.17 & 32.3 \\
\hline $928 \mathrm{~A}-3 \mathrm{H}-5,75-77$ & 26.89 & 20.6 & 2.0 & $928 \mathrm{~B}-4 \mathrm{H}-3,82-83$ & 31.22 & 18.0 \\
\hline $928 \mathrm{~A}-3 \mathrm{H}-5,80-82$ & 26.94 & 23.1 & 3.3 & $928 \mathrm{~B}-4 \mathrm{H}-3,87-88$ & 31.27 & 12.2 \\
\hline $928 \mathrm{~A}-3 \mathrm{H}-5,85-87$ & 26.99 & 31.1 & 6.8 & $928 \mathrm{~B}-4 \mathrm{H}-3,92-93$ & 31.32 & 10.5 \\
\hline $928 \mathrm{~A}-3 \mathrm{H}-5,90-91$ & 27.04 & & 9.8 & $928 \mathrm{~B}-4 \mathrm{H}-3,97-98$ & 31.37 & 9.9 \\
\hline $928 \mathrm{~A}-3 \mathrm{H}-5,95-96$ & 27.09 & & 10.4 & $928 \mathrm{~B}-4 \mathrm{H}-3,102-103$ & 31.42 & 14.4 \\
\hline $928 \mathrm{~A}-3 \mathrm{H}-5,100-101$ & 27.14 & & 12.2 & $928 \mathrm{~B}-4 \mathrm{H}-3,107-108$ & 31.47 & 13.8 \\
\hline 928A-3H-5, 105-106 & 27.19 & & 12.2 & $928 \mathrm{~B}-4 \mathrm{H}-3,112-113$ & 31.52 & 18.2 \\
\hline $928 \mathrm{~A}-3 \mathrm{H}-5,110-111$ & 27.24 & & 14.4 & $928 \mathrm{~B}-4 \mathrm{H}-3,117-118$ & 31.57 & 25.1 \\
\hline $928 \mathrm{~A}-3 \mathrm{H}-5,115-116$ & 27.29 & 36.5 & 13.8 & $928 \mathrm{~B}-4 \mathrm{H}-3,122-123$ & 31.62 & 30.9 \\
\hline $928 \mathrm{~A}-3 \mathrm{H}-5,120-121$ & 27.34 & & 13.2 & $928 \mathrm{~B}-4 \mathrm{H}-3,127-128$ & 31.67 & 19.3 \\
\hline $928 \mathrm{~A}-3 \mathrm{H}-5,125-126$ & 27.39 & 37.5 & 13.0 & $928 \mathrm{~B}-4 \mathrm{H}-3,132-133$ & 31.72 & 16.6 \\
\hline $928 \mathrm{~A}-3 \mathrm{H}-5,130-131$ & 27.44 & 35.0 & 10.0 & $928 \mathrm{~B}-4 \mathrm{H}-3,137-138$ & 31.77 & 11.6 \\
\hline $928 \mathrm{~A}-3 \mathrm{H}-5,135-136$ & 27.49 & 35.0 & 10.1 & $928 \mathrm{~B}-4 \mathrm{H}-3,142-143$ & 31.82 & 11.7 \\
\hline $928 \mathrm{~A}-3 \mathrm{H}-5,140-141$ & 27.54 & 41.9 & 11.5 & $928 \mathrm{~B}-4 \mathrm{H}-3,147-148$ & 31.87 & 17.4 \\
\hline 928A-3H-5, 145-146 & 27.59 & 35.6 & 9.8 & $928 \mathrm{~B}-4 \mathrm{H}-4,2-3$ & 31.92 & 30.7 \\
\hline $928 \mathrm{~A}-3 \mathrm{H}-6,0-2$ & 27.64 & 37.8 & 11.0 & $928 \mathrm{~B}-4 \mathrm{H}-4,7-8$ & 31.97 & 32.0 \\
\hline $928 \mathrm{~A}-3 \mathrm{H}-6,5-7$ & 27.69 & 35.8 & $\begin{array}{r}11.0 \\
9.9\end{array}$ & $928 \mathrm{~B}-4 \mathrm{H}-4,12-13$ & 32.02 & 36.2 \\
\hline $928 \mathrm{~A}-3 \mathrm{H}-6,10-12$ & 27.74 & 26.7 & 6.6 & $928 \mathrm{~B}-4 \mathrm{H}-4,17-18$ & 32.07 & 36.4 \\
\hline $928 \mathrm{~A}-3 \mathrm{H}-6,15-17$ & 27.79 & 17.5 & 1.4 & $928 \mathrm{~B}-4 \mathrm{H}-4,22-23$ & 32.12 & 35.5 \\
\hline $928 \mathrm{~A}-3 \mathrm{H}-6,20-22$ & 27.84 & 9.2 & 2.3 & $928 \mathrm{~B}-4 \mathrm{H}-4,27-28$ & 32.17 & 34.4 \\
\hline $928 \mathrm{~A}-3 \mathrm{H}-6,25-27$ & 27.89 & 16.7 & 1.2 & $928 \mathrm{~B}-4 \mathrm{H}-4,32-33$ & 32.22 & 34.9 \\
\hline $928 \mathrm{~A}-3 \mathrm{H}-6,30-32$ & 27.94 & 17.8 & 1.2 & $928 \mathrm{~B}-4 \mathrm{H}-4,37-38$ & 32.27 & 40.3 \\
\hline $928 \mathrm{~A}-3 \mathrm{H}-6,35-37$ & 27.99 & 17.1 & 0.7 & $928 \mathrm{~B}-4 \mathrm{H}-4,42-43$ & 32.32 & 38.9 \\
\hline $928 \mathrm{~A}-3 \mathrm{H}-6,40-42$ & 28.04 & 18.8 & 1.8 & $928 \mathrm{~B}-4 \mathrm{H}-4,47-48$ & 32.37 & 36.9 \\
\hline $928 \mathrm{~A}-3 \mathrm{H}-6,45-47$ & 28.09 & 22.1 & 1.4 & $928 \mathrm{~B}-4 \mathrm{H}-4,52-53$ & 32.42 & 25.3 \\
\hline $928 \mathrm{~A}-3 \mathrm{H}-6,50-52$ & 28.14 & 21.0 & 0.7 & $928 \mathrm{~B}-4 \mathrm{H}-4,57-58$ & 32.47 & 17.6 \\
\hline $928 \mathrm{~A}-3 \mathrm{H}-6,55-57$ & 28.19 & 22.5 & 1.3 & $928 \mathrm{~B}-4 \mathrm{H}-4,62-63$ & 32.52 & 14.8 \\
\hline $928 \mathrm{~A}-3 \mathrm{H}-6,60-62$ & 28.24 & 26.8 & 2.7 & $928 \mathrm{~B}-4 \mathrm{H}-4,67-68$ & 32.57 & 15.3 \\
\hline $928 \mathrm{~A}-3 \mathrm{H}-6,65-67$ & 28.29 & 16.3 & 0.8 & $928 \mathrm{~B}-4 \mathrm{H}-4,72-73$ & 32.62 & 16.2 \\
\hline $928 \mathrm{~A}-3 \mathrm{H}-6,70-72$ & 28.34 & 18.5 & 1.4 & $928 \mathrm{~B}-4 \mathrm{H}-4,77-78$ & 32.67 & 16.5 \\
\hline $928 \mathrm{~A}-3 \mathrm{H}-6,75-77$ & 28.39 & 21.0 & 1.2 & $928 \mathrm{~B}-4 \mathrm{H}-4,82-83$ & 32.72 & 21.3 \\
\hline $928 \mathrm{~A}-3 \mathrm{H}-6,80-82$ & 28.44 & 22.4 & 2.6 & $928 \mathrm{~B}-4 \mathrm{H}-4,87-88$ & 32.77 & 26.4 \\
\hline $928 \mathrm{~A}-3 \mathrm{H}-6,85-87$ & 28.49 & 18.2 & 1.2 & $928 \mathrm{~B}-4 \mathrm{H}-4,92-93$ & 32.82 & 8.5 \\
\hline $928 \mathrm{~A}-3 \mathrm{H}-6,90-91$ & 28.54 & & 0.8 & 928B-4H-4, 97-98 & 32.87 & 11.9 \\
\hline $928 \mathrm{~A}-3 \mathrm{H}-6,95-96$ & 28.59 & & 1.7 & $928 \mathrm{~B}-4 \mathrm{H}-4,102-103$ & 32.92 & 11.4 \\
\hline
\end{tabular}


Table 2 (continued).

\begin{tabular}{|c|c|c|c|}
\hline $\begin{array}{l}\text { Hole, core, section, } \\
\text { interval }(\mathrm{cm})\end{array}$ & $\begin{array}{l}\text { Depth } \\
\text { (mcd) }\end{array}$ & $\begin{array}{c}\mathrm{CaCO}_{3} \\
(\%)\end{array}$ & $\begin{array}{c}>63 \mu \mathrm{m} \\
(\%)\end{array}$ \\
\hline $928 \mathrm{~B}-4 \mathrm{H}-4,107-108$ & 32.97 & 11.9 & 1.9 \\
\hline $928 \mathrm{~B}-4 \mathrm{H}-4,112-113$ & 33.02 & 21.3 & 1.8 \\
\hline $928 \mathrm{~B}-4 \mathrm{H}-4,117-118$ & 33.07 & 11.6 & 0.9 \\
\hline $928 \mathrm{~B}-4 \mathrm{H}-4,122-123$ & 33.12 & 36.6 & 1.1 \\
\hline $928 \mathrm{~B}-4 \mathrm{H}-4,127-128$ & 33.17 & 26.1 & 1.7 \\
\hline $928 \mathrm{~B}-4 \mathrm{H}-4,132-133$ & 33.22 & 22.2 & 1.1 \\
\hline $928 \mathrm{~B}-4 \mathrm{H}-4,137-138$ & 33.27 & 34.7 & 1.6 \\
\hline $928 \mathrm{~B}-4 \mathrm{H}-4,142-143$ & 33.32 & 34.9 & 5.0 \\
\hline $928 \mathrm{~B}-4 \mathrm{H}-4,147-148$ & 33.37 & 45.7 & 11.3 \\
\hline $928 \mathrm{~B}-4 \mathrm{H}-5,2-3$ & 33.42 & 48.6 & 13.4 \\
\hline $928 \mathrm{~B}-4 \mathrm{H}-5,7-8$ & 33.47 & 54.5 & 18.2 \\
\hline $928 \mathrm{~B}-4 \mathrm{H}-5,12-13$ & 33.52 & 59.4 & 16.9 \\
\hline $928 \mathrm{~B}-4 \mathrm{H}-5,17-18$ & 33.57 & 50.6 & 5.5 \\
\hline $928 \mathrm{~B}-4 \mathrm{H}-5,22-23$ & 33.62 & 47.2 & 3.8 \\
\hline $928 \mathrm{~B}-4 \mathrm{H}-5,27-28$ & 33.67 & 13.9 & 0.6 \\
\hline $928 \mathrm{~B}-4 \mathrm{H}-5,32-33$ & 33.72 & 29.6 & 1.3 \\
\hline $928 \mathrm{~B}-4 \mathrm{H}-5,37-38$ & 33.77 & 33.5 & 0.2 \\
\hline 928B-4H-5, 42-43 & 33.82 & 34.7 & 2.2 \\
\hline $928 \mathrm{~B}-4 \mathrm{H}-5,47-48$ & 33.87 & 32.4 & 0.1 \\
\hline $928 \mathrm{~B}-4 \mathrm{H}-5,52-53$ & 33.92 & 32.4 & 3.0 \\
\hline $928 \mathrm{~B}-4 \mathrm{H}-5,57-58$ & 33.97 & 37.5 & 0.3 \\
\hline $928 \mathrm{~B}-4 \mathrm{H}-5,62-63$ & 34.02 & 38.9 & 5.3 \\
\hline $928 \mathrm{~B}-4 \mathrm{H}-5,67-68$ & 34.07 & 35.2 & 0.2 \\
\hline $928 \mathrm{~B}-4 \mathrm{H}-5,72-73$ & 34.12 & 44.9 & 5.9 \\
\hline $928 \mathrm{~B}-4 \mathrm{H}-5,77-78$ & 34.17 & 39.8 & 0.4 \\
\hline $928 \mathrm{~B}-4 \mathrm{H}-5,82-83$ & 34.22 & 32.7 & $\begin{array}{l}0.4 \\
2.7\end{array}$ \\
\hline $928 \mathrm{~B}-4 \mathrm{H}-5,87-88$ & 34.27 & 31.3 & 0.1 \\
\hline 928B-4H-5, 92-93 & 34.32 & 34.8 & 1.8 \\
\hline 928B-4H-5, $97-98$ & 34.37 & 23.3 & 0.1 \\
\hline $928 \mathrm{~B}-4 \mathrm{H}-5,102-103$ & 34.42 & 25.6 & 3.1 \\
\hline $928 \mathrm{~B}-4 \mathrm{H}-5,107-108$ & 34.47 & 28.7 & 0.2 \\
\hline $928 \mathrm{~B}-4 \mathrm{H}-5,112-113$ & 34.52 & 34.7 & 3.7 \\
\hline $928 \mathrm{~B}-4 \mathrm{H}-5,117-118$ & 34.57 & 38.4 & 0.3 \\
\hline $928 \mathrm{C}-4 \mathrm{H}-2,2-3$ & 34.58 & 47.8 & 9.7 \\
\hline $928 \mathrm{~B}-4 \mathrm{H}-5,122-123$ & 34.62 & 50.0 & 9.1 \\
\hline $928 \mathrm{C}-4 \mathrm{H}-2,7-8$ & 34.63 & 45.3 & 9.6 \\
\hline $928 \mathrm{~B}-4 \mathrm{H}-5,127-128$ & 34.67 & 49.5 & 11.1 \\
\hline $928 \mathrm{C}-4 \mathrm{H}-2,12-13$ & 34.68 & 36.9 & 7.9 \\
\hline $928 \mathrm{C}-4 \mathrm{H}-2,17-18$ & 34.73 & 29.7 & 4.7 \\
\hline $928 \mathrm{C}-4 \mathrm{H}-2,22-23$ & 34.78 & 20.8 & 2.4 \\
\hline $928 \mathrm{C}-4 \mathrm{H}-2,27-28$ & 34.83 & 22.5 & 3.0 \\
\hline $928 \mathrm{C}-4 \mathrm{H}-2,32-33$ & 34.88 & 20.0 & 1.9 \\
\hline $928 \mathrm{C}-4 \mathrm{H}-2,37-38$ & 34.93 & 18.1 & 1.7 \\
\hline $928 \mathrm{C}-4 \mathrm{H}-2,42-43$ & 34.98 & 15.3 & 1.3 \\
\hline $928 \mathrm{C}-4 \mathrm{H}-2,47-48$ & 35.03 & 16.1 & 1.4 \\
\hline $928 \mathrm{C}-4 \mathrm{H}-2,52-53$ & 35.08 & 23.3 & $\begin{array}{l}2.8 \\
2.8\end{array}$ \\
\hline $928 \mathrm{C}-4 \mathrm{H}-2,57-58$ & 35.13 & 23.1 & 3.8 \\
\hline $928 \mathrm{C}-4 \mathrm{H}-2,62-63$ & 35.18 & 16.1 & 1.4 \\
\hline $928 \mathrm{C}-4 \mathrm{H}-2,67-68$ & 35.23 & 22.1 & $\begin{array}{l}2.4 \\
2.5\end{array}$ \\
\hline $928 \mathrm{C}-4 \mathrm{H}-2,72-73$ & 35.28 & 46.1 & 11.7 \\
\hline $928 \mathrm{C}-4 \mathrm{H}-2,77-78$ & 35.33 & 50.0 & 14.0 \\
\hline $928 \mathrm{C}-4 \mathrm{H}-2,82-83$ & 35.38 & 48.9 & $\begin{array}{l}16.1 \\
16.1\end{array}$ \\
\hline $928 \mathrm{C}-4 \mathrm{H}-2,87-88$ & 35.43 & 43.9 & 6.1 \\
\hline $928 \mathrm{C}-4 \mathrm{H}-2,92-93$ & 35.48 & 36.9 & 10.1 \\
\hline $928 \mathrm{C}-4 \mathrm{H}-2,97-98$ & 35.53 & 33.6 & $\begin{array}{r}10.1 \\
8.2\end{array}$ \\
\hline $928 \mathrm{C}-4 \mathrm{H}-2,102-103$ & 35.58 & 37.8 & 8.4 \\
\hline $928 \mathrm{C}-4 \mathrm{H}-2,107-108$ & 35.63 & 36.4 & 10.3 \\
\hline$-4 \mathrm{H}-2,112-113$ & 35.68 & 37.8 & 10.5 \\
\hline $928 \mathrm{C}-4 \mathrm{H}-2,117-118$ & 35.73 & 36.7 & 10.1 \\
\hline $928 \mathrm{C}-4 \mathrm{H}-2,122-123$ & 35.78 & 38.1 & 11.6 \\
\hline $928 \mathrm{C}-4 \mathrm{H}-2,127-128$ & 35.83 & 33.9 & 9.4 \\
\hline $928 \mathrm{C}-4 \mathrm{H}-2,132-133$ & 35.88 & 30.3 & 7.8 \\
\hline $928 \mathrm{C}-4 \mathrm{H}-2,137-138$ & 35.93 & 25.0 & $\because 0$ \\
\hline $4 \mathrm{H}-2,142-143$ & 35 . & 19.7 & 2.2 \\
\hline $928 \mathrm{C}-4 \mathrm{H}-2,147-148$ & 36.03 & 15.3 & 1.4 \\
\hline $928 \mathrm{C}-4 \mathrm{H}-3,2-3$ & 36.08 & 13.1 & 1.1 \\
\hline $928 \mathrm{C}-4 \mathrm{H}-3,7-8$ & 36.13 & 11.4 & $\begin{array}{l}1.1 \\
1.0\end{array}$ \\
\hline S & 2618 & 6 & 0.4 \\
\hline $4 \mathrm{H}-3$ & 36.23 & 6.7 & 0.6 \\
\hline
\end{tabular}

\begin{tabular}{|c|c|c|c|}
\hline $\begin{array}{l}\text { Hole, core, section, } \\
\text { interval }(\mathrm{cm})\end{array}$ & $\begin{array}{l}\text { Depth } \\
\text { (mcd) }\end{array}$ & $\begin{array}{c}\mathrm{CaCO}_{3} \\
(\%)\end{array}$ & $\begin{array}{l}>63 \mu \mathrm{m} \\
(\%)\end{array}$ \\
\hline $928 \mathrm{C}-4 \mathrm{H}-3,22-23$ & 36.28 & 10.8 & 1.2 \\
\hline $928 \mathrm{C}-4 \mathrm{H}-3,27-28$ & 36.33 & 15.6 & 4.8 \\
\hline $928 \mathrm{C}-4 \mathrm{H}-3,32-33$ & 36.38 & 40.6 & 13.3 \\
\hline $928 \mathrm{C}-4 \mathrm{H}-3,37-38$ & 36.43 & 47.5 & 19.2 \\
\hline $928 \mathrm{C}-4 \mathrm{H}-3,42-43$ & 36.48 & 53.1 & 21.7 \\
\hline $928 \mathrm{C}-4 \mathrm{H}-3,47-48$ & 36.53 & 61.7 & 25.3 \\
\hline $928 \mathrm{C}-4 \mathrm{H}-3,52-53$ & 36.58 & 59.9 & 25.3 \\
\hline $928 \mathrm{C}-4 \mathrm{H}-3,57-58$ & 36.63 & 51.1 & 22.3 \\
\hline $928 \mathrm{C}-4 \mathrm{H}-3,62-63$ & 36.68 & 40.0 & 16.1 \\
\hline $928 \mathrm{C}-4 \mathrm{H}-3,67-68$ & 36.73 & 40.6 & 18.6 \\
\hline $928 \mathrm{C}-4 \mathrm{H}-3,72-73$ & 36.78 & 37.2 & 17.0 \\
\hline $928 \mathrm{C}-4 \mathrm{H}-3,77-78$ & 36.83 & 34.2 & 16.9 \\
\hline $928 \mathrm{C}-4 \mathrm{H}-3,82-83$ & 36.88 & 34.4 & 16.6 \\
\hline $928 \mathrm{C}-4 \mathrm{H}-3,87-88$ & 36.93 & 31.1 & 14.1 \\
\hline $928 \mathrm{C}-4 \mathrm{H}-3,92-93$ & 36.98 & 32.8 & 11.9 \\
\hline $928 \mathrm{C}-4 \mathrm{H}-3,97-98$ & 37.03 & 34.4 & 11.5 \\
\hline $928 \mathrm{C}-4 \mathrm{H}-3,107-108$ & 37.13 & & 12.8 \\
\hline $928 \mathrm{C}-4 \mathrm{H}-3,117-118$ & 37.23 & & 6.3 \\
\hline $928 \mathrm{C}-4 \mathrm{H}-3,127-128$ & 37.33 & & 1.2 \\
\hline $928 \mathrm{C}-4 \mathrm{H}-3,137-138$ & 37.43 & & 1.6 \\
\hline $928 \mathrm{C}-4 \mathrm{H}-3,147-148$ & 37.53 & & 2.9 \\
\hline $928 \mathrm{C}-4 \mathrm{H}-4,2-3$ & 37.58 & & 13.0 \\
\hline $928 \mathrm{C}-4 \mathrm{H}-4,7-8$ & 37.63 & & 18.0 \\
\hline $928 \mathrm{C}-4 \mathrm{H}-4,17-18$ & 37.73 & & 18.6 \\
\hline $928 \mathrm{C}-4 \mathrm{H}-4,27-28$ & 37.83 & & 12.5 \\
\hline $928 \mathrm{C}-4 \mathrm{H}-4,37-38$ & 37.93 & & 11.1 \\
\hline $928 \mathrm{C}-4 \mathrm{H}-4,47-48$ & 38.03 & & 2.7 \\
\hline $928 \mathrm{C}-4 \mathrm{H}-4,57-58$ & 38.13 & & 1.8 \\
\hline $928 \mathrm{C}-4 \mathrm{H}-4,67-68$ & 38.23 & & 3.7 \\
\hline $928 \mathrm{C}-4 \mathrm{H}-4,77-78$ & 38.33 & & 3.2 \\
\hline $928 \mathrm{C}-4 \mathrm{H}-4,87-88$ & 38.43 & & 3.6 \\
\hline $928 \mathrm{C}-4 \mathrm{H}-4,92-93$ & 38.48 & & 1.2 \\
\hline $928 \mathrm{C}-4 \mathrm{H}-4,97-98$ & 38.53 & & 1.4 \\
\hline $928 \mathrm{C}-4 \mathrm{H}-4,107-108$ & 38.63 & & 1.8 \\
\hline $928 \mathrm{C}-4 \mathrm{H}-4,117-118$ & 38.73 & & 5.8 \\
\hline $928 \mathrm{C}-4 \mathrm{H}-4,127-128$ & 38.83 & & 3.9 \\
\hline $928 \mathrm{C}-4 \mathrm{H}-4,137-138$ & 38.93 & & 9.3 \\
\hline $928 \mathrm{C}-4 \mathrm{H}-4,147-148$ & 39.03 & & 15.6 \\
\hline $928 \mathrm{C}-4 \mathrm{H}-5,7-8$ & 39.13 & & 23.0 \\
\hline $928 \mathrm{C}-4 \mathrm{H}-5,17-18$ & 39.23 & & 16.1 \\
\hline $928 \mathrm{C}-4 \mathrm{H}-5,27-28$ & 39.33 & & 10.1 \\
\hline $928 \mathrm{C}-4 \mathrm{H}-5,37-38$ & 39.43 & & 9.5 \\
\hline $928 \mathrm{C}-4 \mathrm{H}-5,47-48$ & 39.53 & & 9.4 \\
\hline $928 \mathrm{C}-4 \mathrm{H}-5,57-58$ & 39.63 & & 10.7 \\
\hline $928 \mathrm{C}-4 \mathrm{H}-5,67-68$ & 39.73 & & 9.7 \\
\hline $928 \mathrm{C}-4 \mathrm{H}-5,77-78$ & 39.83 & & 12.9 \\
\hline $928 \mathrm{C}-4 \mathrm{H}-5,87-88$ & 39.93 & & 17.6 \\
\hline $928 \mathrm{C}-4 \mathrm{H}-5,97-98$ & 40.03 & & 16.8 \\
\hline $928 \mathrm{C}-4 \mathrm{H}-5,107-108$ & 40.13 & & 10.1 \\
\hline $928 \mathrm{C}-4 \mathrm{H}-5,117-118$ & 40.23 & & 7.3 \\
\hline $928 \mathrm{C}-4 \mathrm{H}-5,127-128$ & 40.33 & & 4.3 \\
\hline $928 \mathrm{C}-4 \mathrm{H}-5,132-133$ & 40.38 & & 6.7 \\
\hline $928 \mathrm{C}-4 \mathrm{H}-5,137-138$ & 40.43 & & 5.6 \\
\hline $928 \mathrm{C}-4 \mathrm{H}-5,147-148$ & 40.53 & & 2.8 \\
\hline $928 \mathrm{C}-4 \mathrm{H}-6,7-8$ & 40.63 & & 3.7 \\
\hline $928 \mathrm{C}-4 \mathrm{H}-6,17-18$ & 40.73 & & 2.7 \\
\hline $928 \mathrm{C}-4 \mathrm{H}-6,27-28$ & 40.83 & & 5.4 \\
\hline $928 \mathrm{C}-4 \mathrm{H}-6,37-38$ & 40.93 & & 0.9 \\
\hline $928 \mathrm{C}-4 \mathrm{H}-6,47-48$ & 41.03 & & 1.4 \\
\hline $928 \mathrm{C}-4 \mathrm{H}-6,57-58$ & 41.13 & & 2.2 \\
\hline $928 \mathrm{C}-4 \mathrm{H}-6,67-68$ & 41.23 & & 7.1 \\
\hline $928 \mathrm{C}-4 \mathrm{H}-6,77-78$ & 41.33 & & 13.4 \\
\hline $928 \mathrm{C}-4 \mathrm{H}-6,87-88$ & 41.43 & & 13.5 \\
\hline $928 \mathrm{C}-4 \mathrm{H}-6,97-98$ & 41.53 & & 18.5 \\
\hline $928 \mathrm{C}-4 \mathrm{H}-6,107-108$ & 41.63 & & 14.9 \\
\hline $928 \mathrm{C}-4 \mathrm{H}-6,117-118$ & 41.73 & & 4.4 \\
\hline $928 \mathrm{C}-4 \mathrm{H}-6,127-128$ & 41.83 & & 3.5 \\
\hline 928C-4H-6, 137-138 & 41.93 & & 1.3 \\
\hline 928C-4H-6, 147-148 & 42.03 & & 1.8 \\
\hline
\end{tabular}

ries with gaussian pass-band filters centered on 1/41- and 1/21-k.y. ${ }^{-1}$ frequencies.

Filtered records are presented in Figure 10. These records have been normalized to have a zero mean and a standard deviation of $\sigma=$ 1 . We reversed the sign of the $F$. profunda abundance filtered record so that positive excursions in Figure 10 correspond to low $\delta^{18} \mathrm{O}$ values (interglacial/warm periods), high carbonate MAR values (high biogenic productivity), and low $F$. profunda abundances (shallow nutricline depth, high divergence).

\section{Precession-Related Oscillations}

The 21-k.y. ${ }^{-1}$ oscillations draw a surprisingly complex history that is characterized by important changes in the phase relationships between the $\delta^{18} \mathrm{O}, F$. profunda, and carbonate MAR records over the 680- to 1100-ka interval studied (Fig. 10, right). Because precessionrelated oscillations show relatively low-amplitude changes, we cannot totally rule out the possibility that filtering did not successfully extract pure precession-driven signals resulting from the high noise- 


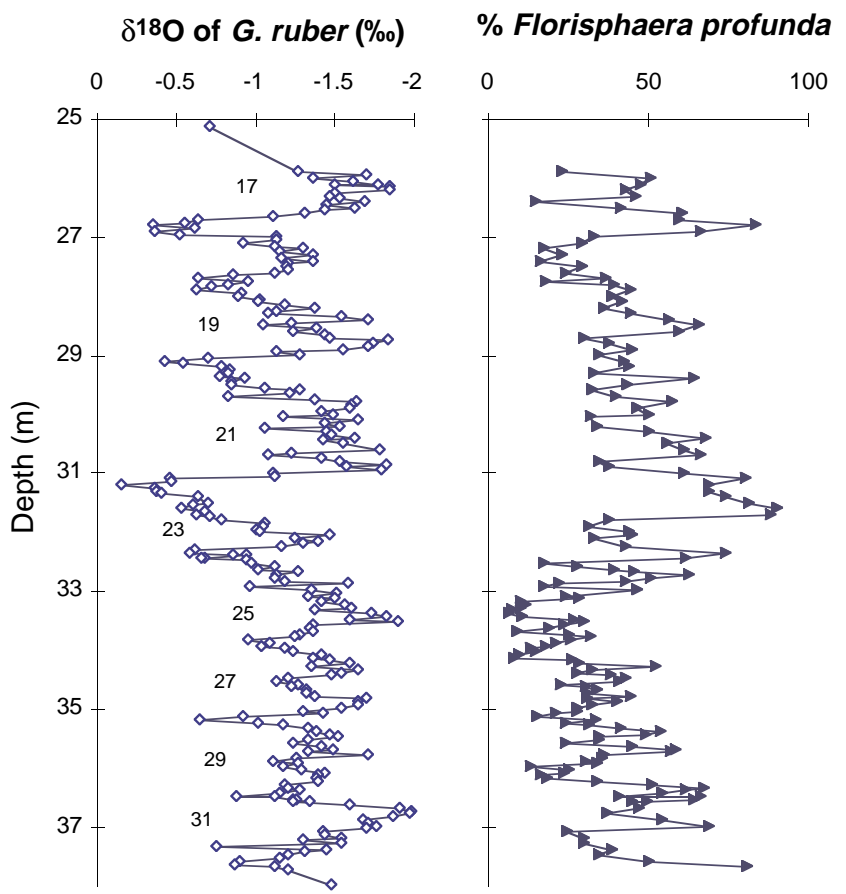

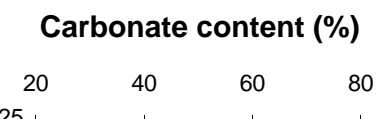

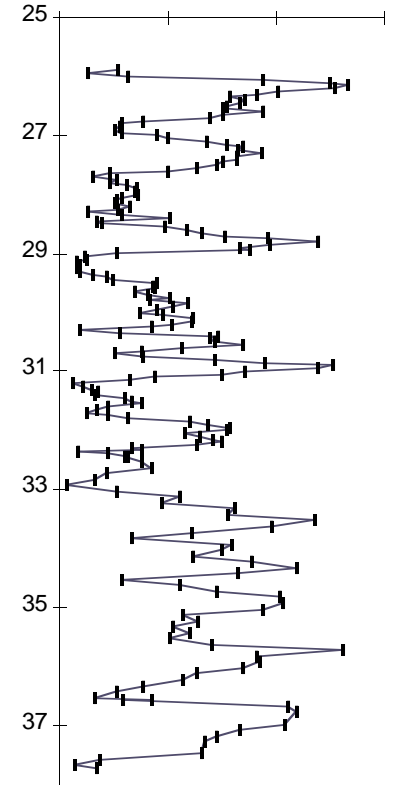

Sand fraction $(>63 \mu \mathrm{m})(\%)$

0

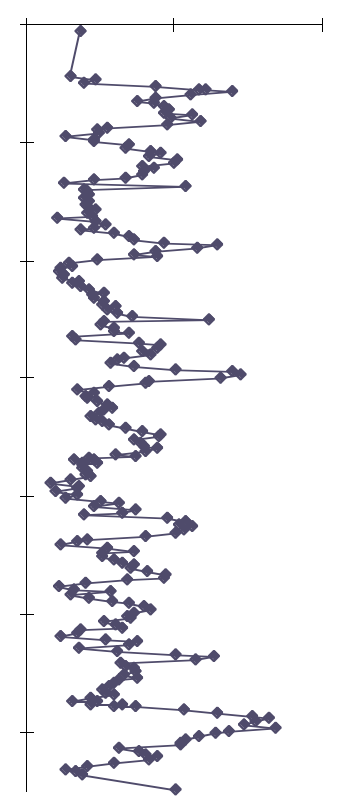

Figure 2. $\delta^{18} \mathrm{O}$ of G. ruber, relative abundance of F. profunda, carbonate content, and sand-sized fraction content vs. depth at Site 925.

\section{Carbonate content (\%)}

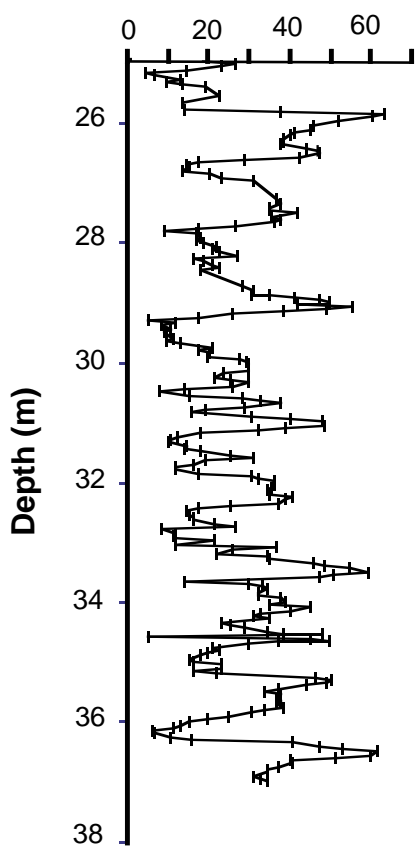

Sand fraction $(>63 \mu \mathrm{m})$

(\%)

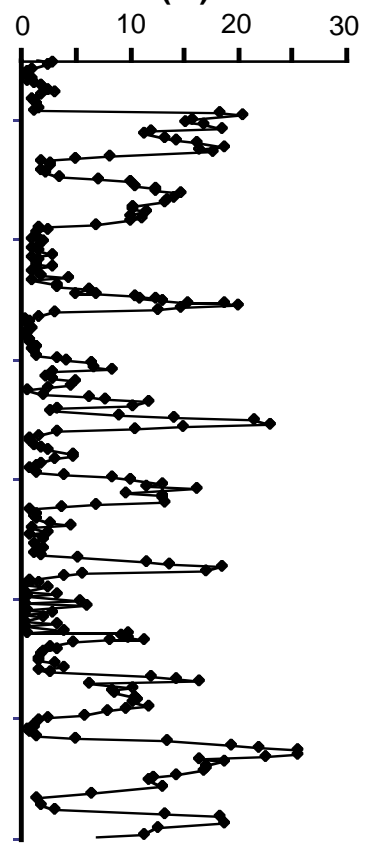

Figure 3. Carbonate content and sand-sized fraction content vs. depth at Site 928.

to-signal ratio of these oscillations. Gaussian filters have a relatively large bandwidth compared to the precession frequencies we tried to extract. Thus, erroneously large or narrow "precession" oscillations may have been obtained over some intervals if noise-related changes were artificially smeared into true precession-driven variations. However, visual examination of the nonfiltered time series tends to confirm the assumption that precession-related oscillations draw a complex history. For instance, a close-up inspection of the unfiltered $\delta^{18} \mathrm{O}$ and $F$. Profunda records in the lower part of the interval studied (between 900 and $1100 \mathrm{ka}$ ), in which all our proxy records have good time resolution, shows that high-frequency changes of these signals have apparently experienced both in-phase and out-of-phase relationships over a relatively short period of time (Fig. 11). It appears also from visual examination of the unfiltered $F$. profunda record that some precession-related changes present a complex "double peak" substructure (Fig. 11).

\section{Obliquity-Related Oscillations}

The 41-k.y. oscillations present a much simpler picture than the 21-k.y. oscillations (Fig. 10, middle). The dominant information that results from filtering our proxy records at $1 / 41 \mathrm{k} . \mathrm{y} .{ }^{-1}$ is the abrupt change in phase relationships observed between about 960 and 920 ka. Prior to $960 \mathrm{ka}$, large phase differences are observed between maxima of the filtered $\delta^{18} \mathrm{O}$, carbonate MAR, and $F$. profunda records. After about $920 \mathrm{ka}$, all the signals have achieved a zerophase relationship. The small ( $<2 \mathrm{k} . \mathrm{y}$.) phase difference observed among $\delta^{18} \mathrm{O}$, carbonate MAR, and $F$. profunda abundance records cannot be considered as statistically robust given the small length of the record available and the inaccuracies related to the filtering. By increasing the length of the interval studied in a future study, we will be able to test the consistency of small leads and lags among the time series.

Our results show that the global, mid-Pleistocene shift in mean and variance of marine $\delta^{18} \mathrm{O}$ records (Maasch, 1988), which occurs roughly between isotopic Stages 25 and 22 (and results in the first severe glaciation of the late Pleistocene), corresponds to a drastic change in phase relationships among low-latitude climatic proxies at Ceara Rise. Stage 24, at about $920 \mathrm{ka}$, is the first isotopic stage in which the climatic proxies have all achieved the zero-phase relationships typical of the upper part of the interval studied.

Prior to $960 \mathrm{ka}$, changes in carbonate-accumulation rates are out of phase with changes in the $F$. profunda record, indicating that 41k.y. changes are most probably driven by regional or global changes in nutrient supplies rather than by local changes in the nutricline dy- 
Figure 4. A. Planktonic $\delta^{18} \mathrm{O}$ records from Site 925 and Site 677 plotted vs. age for comparison (record from Site 677 has been offset by $1 \%$ o for clarity). The synchroneity of isotopic events between these two records tends to confirm that the age model developed at Site 925 is consistent. B. Comparison of $\delta^{18} \mathrm{O}$ record from Site 925 and the ice-volume model of Imbrie and Imbrie (1980) filtered at $1 / 41$ k.y. ${ }^{-1} \mathbf{C}$. Comparison of $\delta^{18} \mathrm{O}$ record from Site 925 and the icevolume model of Imbrie and Imbrie (1980) filtered at $1 / 21 \mathrm{k} . \mathrm{y} \cdot{ }^{-1}$. See text for details.

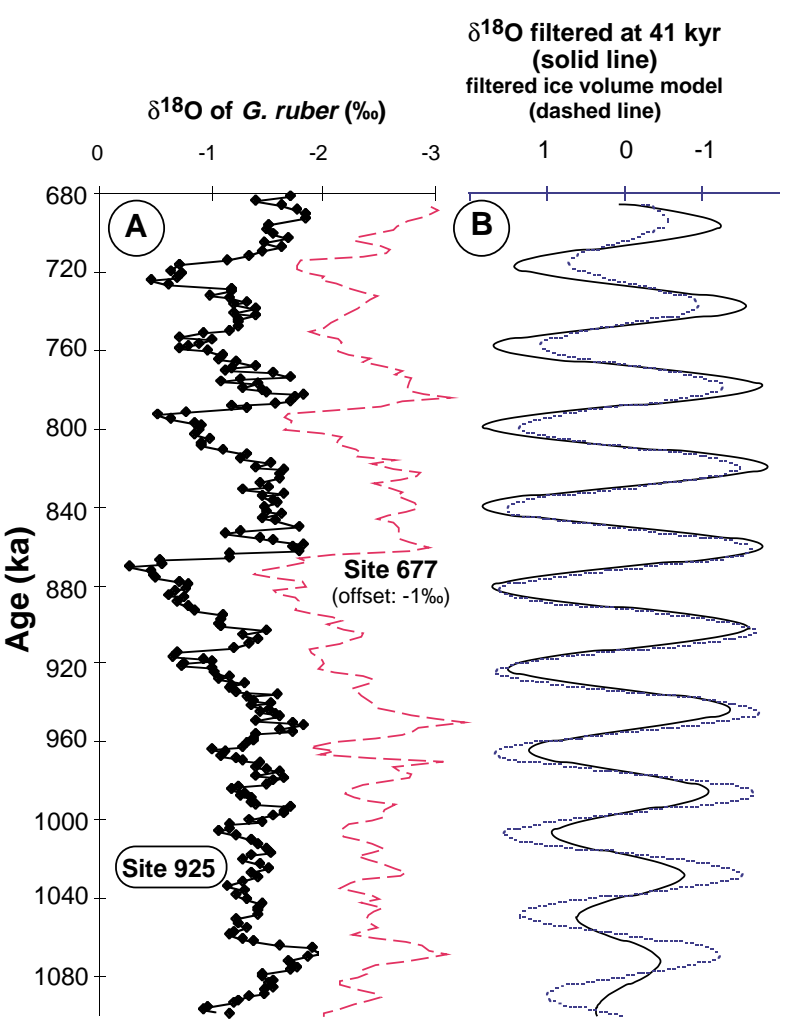

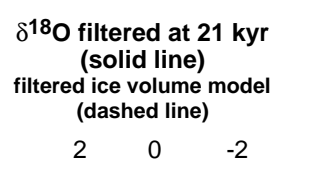
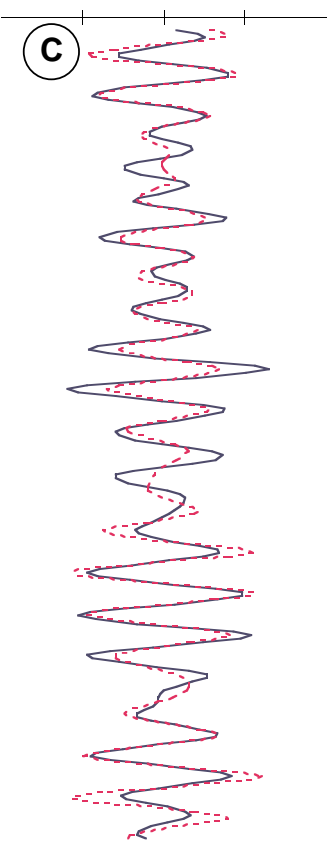

Table 3. Tie-points used for depth-age conversion of sedimentary record from Site 925B.

\begin{tabular}{rr}
\hline $\begin{array}{l}\text { Depth } \\
\text { (mcd) }\end{array}$ & \multicolumn{1}{c}{$\begin{array}{c}\text { Age } \\
\text { (ka) }\end{array}$} \\
\hline 25.94 & 680 \\
26.74 & 718 \\
27.74 & 754 \\
29.09 & 792 \\
30.04 & 831 \\
31.69 & 888 \\
32.57 & 927 \\
33.82 & 964 \\
35.22 & 1006 \\
36.37 & 1056 \\
37.62 & 1098 \\
\end{tabular}

Note: Based on the correlation of the $\delta^{18} \mathrm{O}$ record obtained on Globigerinoides ruber to the ice volume model curve of Imbrie and Imbrie (1980).

namics. After $920 \mathrm{ka}$, maxima in the filtered carbonate MAR record are in phase with minima in $F$. profunda, which indicates that carbonate biogenic productivity is most probably controlled by changes in the nutricline (thermocline) dynamics (Fig. 10).

\section{DISCUSSION}

Today, a large amount of northward, cross-equatorial transport of upper-layer water $(50-100 \mathrm{~m})$ takes place in the western side of the Atlantic Ocean. This cross-equatorial transport of about $7 \mathrm{~Sv}$ of upper waters plays a key role in the return cell of the NADW and contributes heat and salt to the northern high latitudes (Lamb, 1981; Gordon, 1986; Schmitz and McCartney, 1993). We suggest that hydrographic conditions of the western equatorial Atlantic must have played an important role in past changes of Earth's climate, especially those occurring around the "mid-Pleistocene revolution." Because the geographic distribution of major surface currents has a strong imprint on the subsurface topography of the thermocline (see below and Defant,
1961; Hastenrath, 1994), the study of the $F$. profunda changes can provide important information about the evolution of hydrographic conditions on the Ceara Rise over this key period.

Two possible mechanisms may explain past variations in the thermocline structure and in the surface currents on Ceara Rise: 1) changes in trade-wind intensity that would modify the W-E structure of the thermocline topography, and 2) changes in the latitudinal position of the ITCZ (and related atmospheric and hydrographic elements of the low-latitude climatic system) that would result in modifications (with latitudinal shifts?) of the complex, N-S thermocline structure (Fig. 12).

\section{Changes in Trade-Wind Intensity}

In the eastern and central equatorial Atlantic, paleoceanographic studies have suggested that late Pleistocene changes in the thermocline topography were controlled by variations in trade-wind intensity (McIntyre et al., 1989; Molfino and McIntyre, 1990). Increases of trade-wind intensity resulted in increased equatorial divergence and shallowing of the thermocline, whereas weaker trade-wind intensity resulted in reduced equatorial divergence and deepening of the thermocline. No such records of the history of late Quaternary thermocline topography are available for the western equatorial Atlantic. Present oceanographic studies of seasonal hydrographic changes indicate that increases in trade-wind intensity result in the deepening of thermocline in the western equatorial Atlantic because of the piling up of surface waters that are transported from the east (Merle, 1980a, b). If we consider this annual mechanism as an analog for long-term paleoclimatic changes, then it appears that the Ceara Rise should have experienced thermocline responses to trade-wind intensity different from the central and eastern equatorial Atlantic.

During the late Quaternary, trade winds appear to have been strongly controlled by changes in low-latitude insolation. This resulted in the strong 21-k.y. variability observed in the divergence records obtained from analysis of foraminifer and coccolith assemblages (McIntyre et al., 1989; Molfino and McIntyre, 1990). Several global 
$\delta 180$ of G. ruber $(\%)$
\% Florisphaera profunda
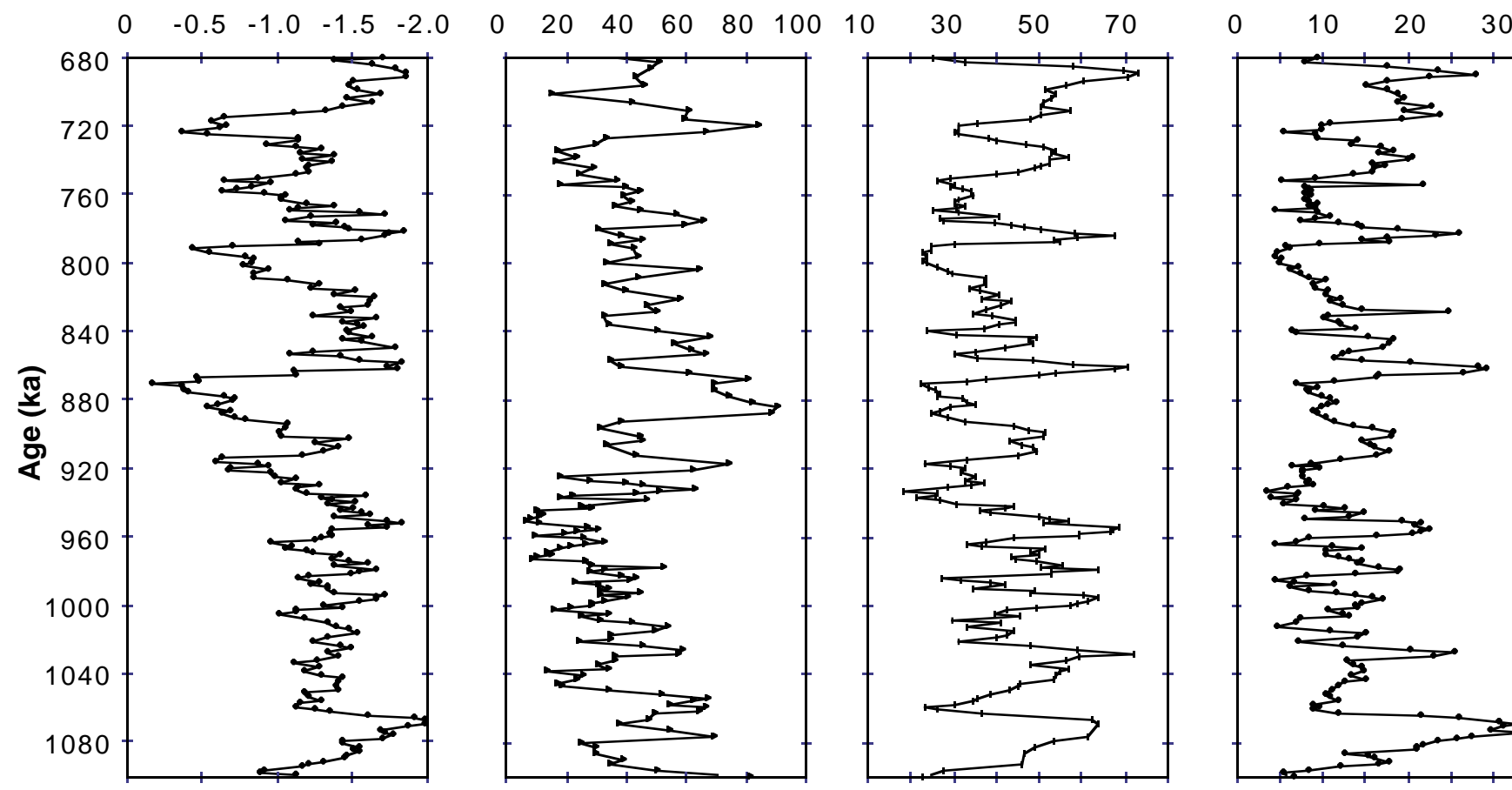

Figure $5 . \delta^{18} \mathrm{O}$ of $G$ r ruber, relative abundance of $F$. profunda, carbonate content, and sand-sized fraction content vs. age at Site 925.

Carbonate content (\%)

\section{Sand fraction (\%)}
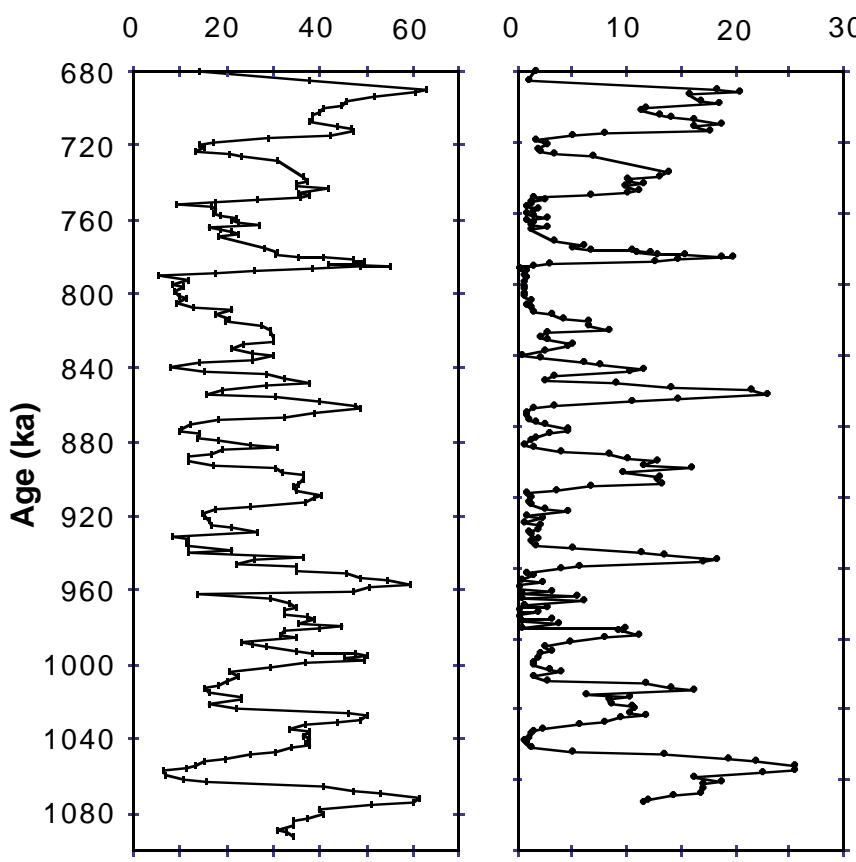

Figure 6. Carbonate content and sand-sized fraction content vs. age at Site 928. climate models and paleoclimatic studies have shown that control of trade winds by low-latitude insolation is related to the intensity of the monsoon over Africa. Tropical easterlies were minimal when perihelion was centered on boreal summer because the North African monsoon was strong and weakened the zonal component of the tropical easterlies (i.e., Manabe and Broccoli, 1985; McIntyre et al., 1989; Molfino and McIntyre, 1990).

Late Quaternary divergence records from the eastern and central equatorial Atlantic also present relatively strong 100-k.y. oscillations that indicate that trade winds are not responding only to direct forcing by low-latitude insolation changes (McIntyre et al., 1989; Molfino and McIntyre, 1990). General circulation model (GCM) studies have shown that cold glacial North Atlantic SSTs result in cooler and drier conditions over North Africa and increased trade-wind speeds (deMenocal and Rind, 1993). Other GCM studies, as well as paleoclimatic reconstructions, have shown that these late Quaternary changes in the north Atlantic SST have been mainly controlled by changes in the volume of the Laurentide ice sheet (Manabe and Broccoli, 1985; Ruddiman and McIntyre, 1984; Ruddiman et al., 1989). The North American ice sheet produces a cold air flow that blows over the North Atlantic and results in cold SST (Manabe and Broccoli, 1985). Over the Pleistocene, the control of North Atlantic SST by changes in the volume of the Laurentide ice sheet is clearly reflected by the good inphase relationship at the 100-k.y. and 41-k.y. periodicities between $\delta^{18} \mathrm{O}$ and SST records measured at Site 607 (Ruddiman et al., 1989). Thus, it appears that the 100-k.y. periodicity observed in late Quaternary divergence records from the equatorial Atlantic could reflect the remote control of trade-wind intensity by changes in North Atlantic SSTs driven by 100-k.y., glacial/interglacial oscillations.

As discussed above, the precession-related signals in the climatic proxy records from Site 925 show an extremely complex history. Assuming that the $F$. profunda signal reflects real paleoclimatic changes, our results would indicate that the relationship between tradewind intensity and thermocline depth may have varied drastically 
Figure 7. Scatter plot of coarse fraction content vs. carbonate content measured from Sites 925 and 928 samples.

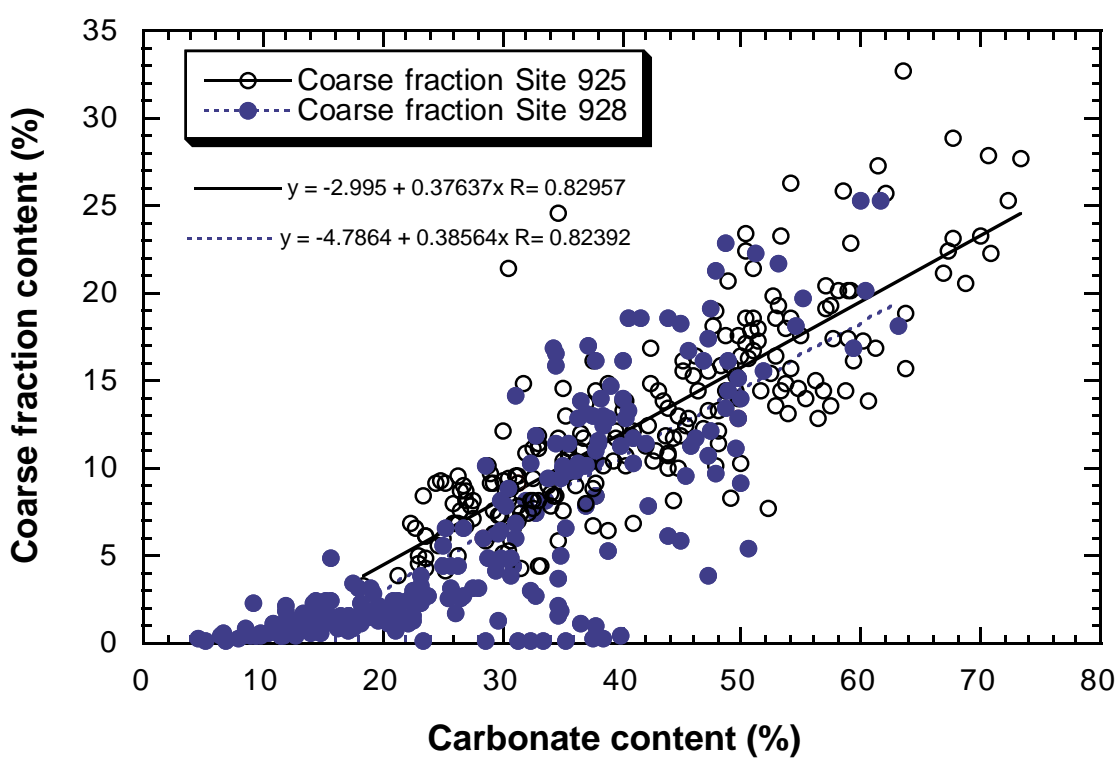

$\delta^{18} \mathrm{O}$ of G. ruber

$(\%)$

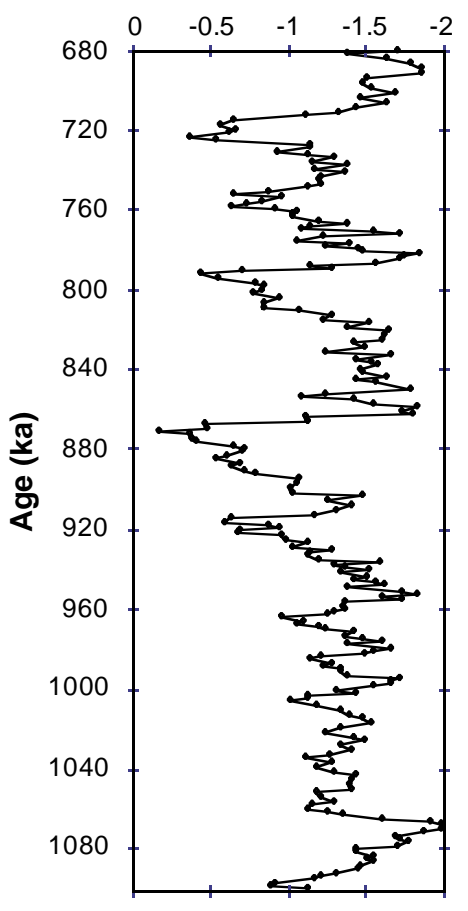

Mass accumulation rates, Site 925 $\left(\mathrm{g} / \mathrm{cm}^{2} / \mathrm{kyr}\right)$

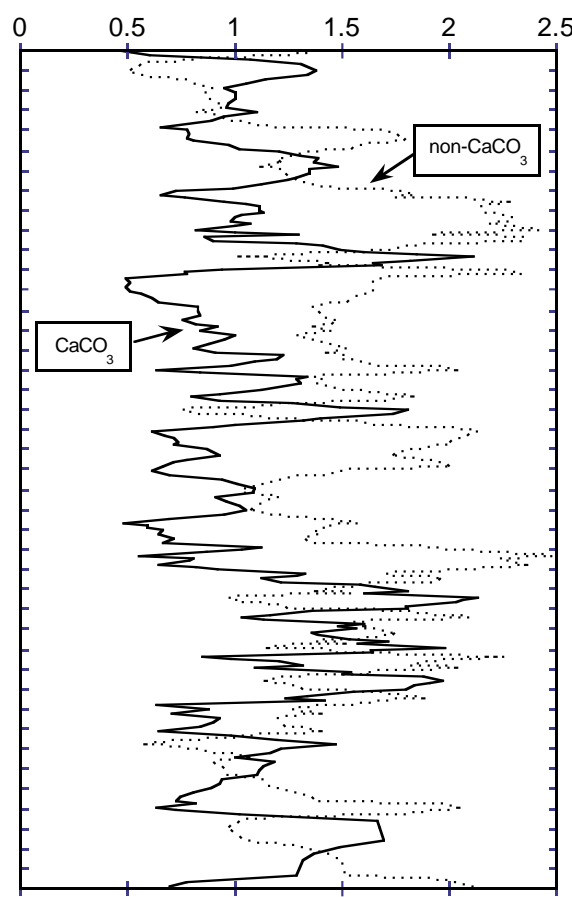

Figure 8. Left: Planktonic $\delta^{18} \mathrm{O}$ record from Site 925 samples. Right: Mass-accumulation rates of carbonate and noncarbonate fractions calculated for Site 925 samples.

The obliquity signals show a more simple and coherent picture over relatively short periods of time $\left(10^{3}-10^{4} \mathrm{yr}\right)$. However, no clear record of equatorial Atlantic trade-wind intensity around $1 \mathrm{Ma}$ exists that we could compare to the $F$. profunda record from Site 925. Long dust records obtained from low-latitude ODP sites situated in the western side of Africa show little (deMenocal, 1995) or even no (Tiedemann et al., 1994) precession-related oscillations around $1 \mathrm{Ma}$. These results could suggest the weak influence of precession-driven insolation changes on trade-wind intensity at this time. Another likely explanation is that these dust records may reflect changes in aridity and dust availability in North Africa rather than changes in the intensity of easterlies (Tiedemann et al., 1994). Given the data available, we are not able to come to a conclusion about precession-driven changes in the western equatorial Atlantic at about $1 \mathrm{Ma}$. than the precession-related signals. Two clearly distinct periods are depicted in the filtered signals, separated by a rather sharp transition interval located between about 960 and 920 k.y. (Fig. 10). We know that changes in the Laurentide ice-sheet volume have controlled 41k.y. changes in North Atlantic SST during the Pleistocene (Ruddiman and McIntyre, 1984; Ruddiman et al., 1989). These SST changes may have exerted a strong control on the zonal intensity of the trade winds by modifying subtropical gyre dynamics. In this scenario, the zerophase relationship observed between the $\delta^{18} \mathrm{O}$ and $F$. profunda records after $920 \mathrm{ka}$ in Site 925 would result from the ice-volume control of the North Atlantic SSTs, which in turn drive the trade-wind intensity and the thermocline dynamics on the Ceara Rise. The rela- 

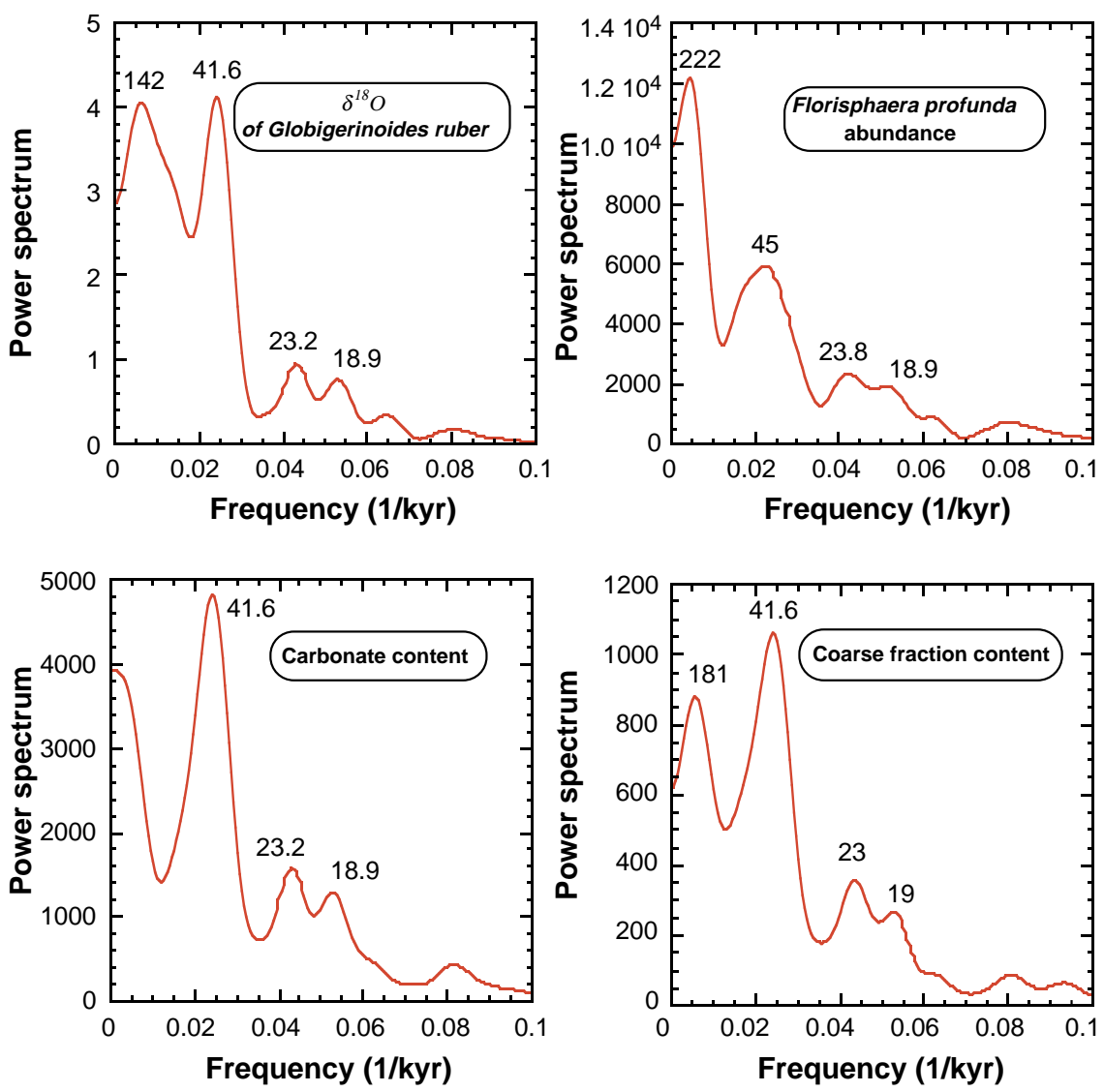

Figure 9. Spectral analysis of planktonic $\delta^{18} \mathrm{O}$ record, F. profunda record, carbonate MAR record, and coarsefraction record obtained from Site 925 samples.

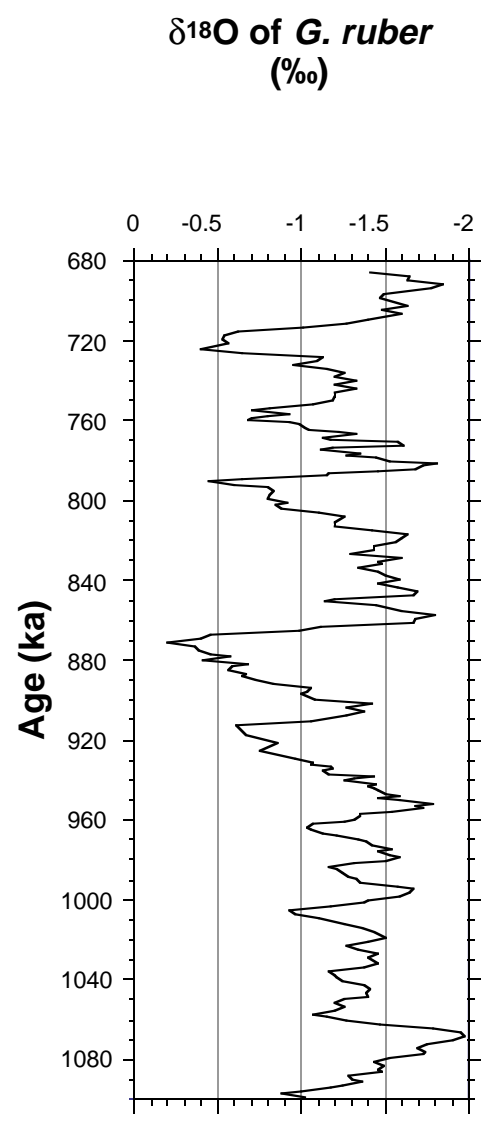

41 kyr oscillations
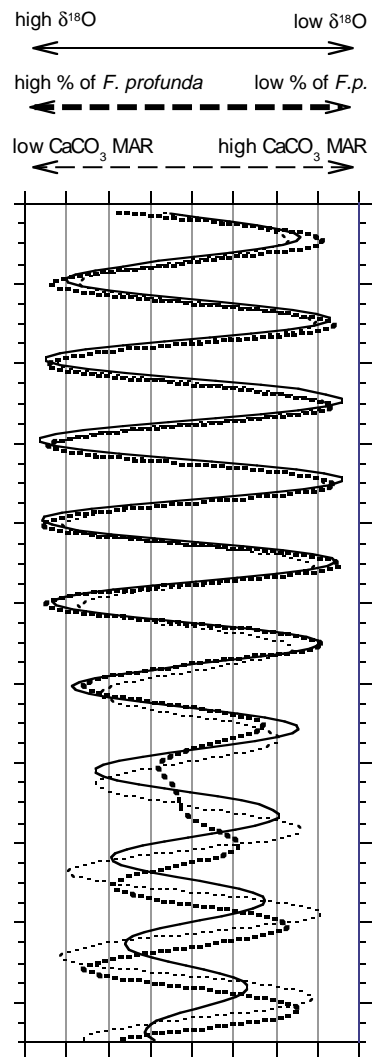

\section{1 kyr oscillations}
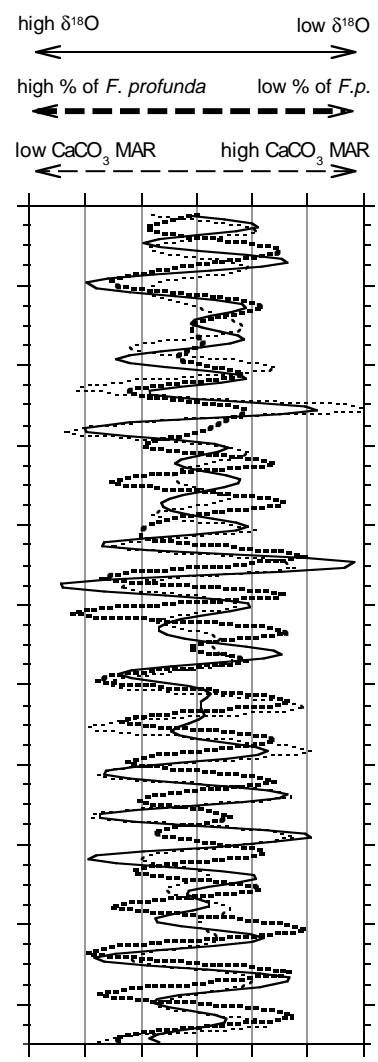

Figure 10. Left: Planktonic $\delta^{18} \mathrm{O}$ record from Site 925. Middle: Comparison of $\delta^{18} \mathrm{O}$, carbonate MAR, and $F$. profunda records filtered at $1 / 41$ k.y. ${ }^{-1}$ Right: Comparison of $\delta^{18} \mathrm{O}$, carbonate MAR, and $F$. profunda records filtered at $1 / 21$ k.y. ${ }^{-1}$ See text for details. 


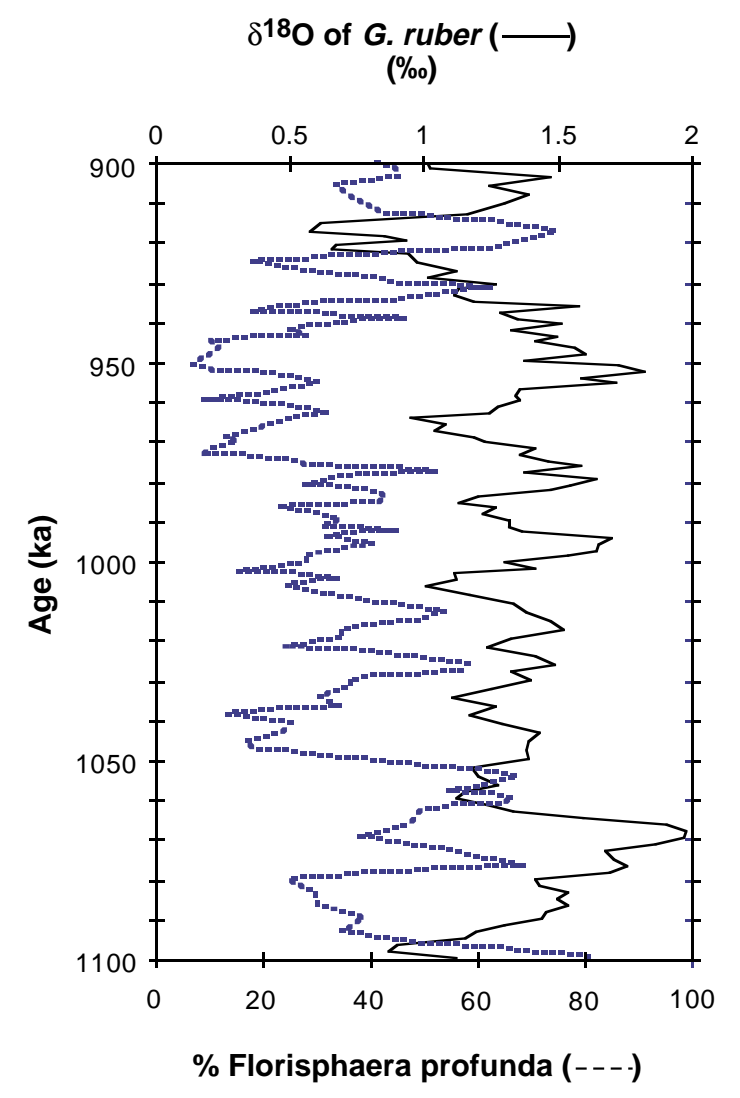

Figure 11. Close-up of $\delta^{18} \mathrm{O}$ and $F$. profunda records from Site 925. Note the complex structure of the high-frequency variations (i.e., double-peak structure of the precession-related oscillations from the $F$. profunda record) and the changes in the phase relationships between the two records. tionship would be as follows: high $\delta^{18} \mathrm{O}$ value (large ice volume) $\rightarrow$ low North Atlantic SSTs $\rightarrow$ high zonal intensity of trade winds $\rightarrow$ deep thermocline upon the Ceara Rise (piling up of surface waters on the west side of the equatorial Atlantic) $\rightarrow$ high F. Profunda abundances. The opposite is true for low $\delta^{18} \mathrm{O}$ values. This scenario is not valid before $960 \mathrm{ka}$, however, because a zero-phase relationship is not achieved between the $\delta^{18} \mathrm{O}$ and the $F$. profunda records. Prior to $960 \mathrm{ka}$, the fact that maxima and minima of the $F$. profunda filtered record occur roughly in the mid-transition between $\delta^{18} \mathrm{O}$ extremes argues strongly against a "delayed" response of any of these signals to changes in the other (we would have to deal with leads and lags of about 10 k.y.). This would imply, therefore, that the "trade-wind" hypothesis is a possible scenario for the period after $920 \mathrm{ka}$ only and may suggest that there exists a threshold ice volume above which ice sheets have an impact on trade-wind intensity.

\section{Changes in the Latitudinal Position of the ITCZ}

The mean annual ITCZ is found today at about $3-5^{\circ} \mathrm{N}$ and results in an asymmetrical structure about the equator of the main westward surface currents that are driven by the trade winds. The main body of the North Equatorial Current (NEC) is located north of $10^{\circ} \mathrm{N}$ and the South Equatorial Current (SEC) extends across the equator. The WE pressure gradient caused by wind-controlled rise in dynamic topography towards the west results in the eastward-flowing North Equatorial Counter Current (NECC) between the NEC and the SEC and at about the same position as the ITCZ (Defant, 1961; Hastenrath, 1994). This asymmetrical structure of the major wind-driven surface currents is reflected in the complex structure of the thermocline topography (Fig. 12; Defant, 1961).

The Ceara Rise is located today in the main body of the NECC, in an area characterized by one of the deepest thermoclines of the western equatorial Atlantic (Fig. 12). Given the strong N-S gradients in thermocline topography, small changes in the mean annual position of the ITCZ, and of related changes in the position of the NEC, NECC, and SEC could have had major effects on the thermocline depth on the Ceara Rise area.
Figure 12. North-south cross-section of the equatorial Atlantic showing the complex structure of the thermocline topography and the position of Site 925 . ITCZ = Intertropical Convergence Zone, NEC = North Equatorial Current, NECC $=$ North Equatorial Counter Current, and SEC $=$ South Equatorial Current (modified from Defant, 1961).

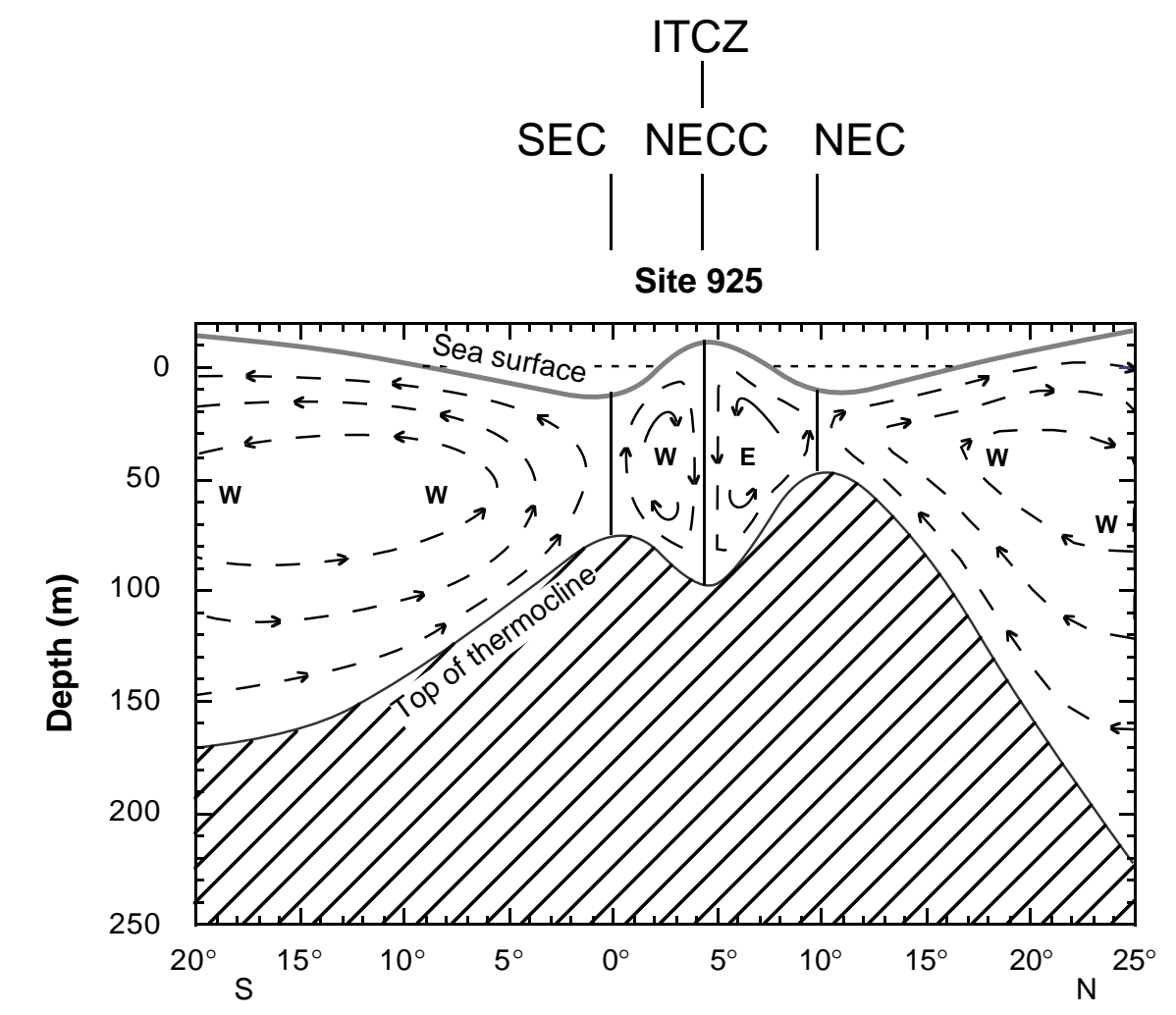

Site 925 
Today, good meteorological and oceanographic evidence supports the seasonal N-S movements of the ITCZ. However, the possibility that changes in the mean annual position of the ITCZ occurred during the Pleistocene is still a matter of debate. Most of the paleoclimatic data available come from the eastern equatorial Atlantic. Based on eolian dust records obtained from sediment cores collected off the west coast of Africa, Sarnthein et al. (1981) concluded that the ITCZ was essentially at the same position at the Last Glacial Maximum (LGM) as today. Based on the long dust record from ODP Site 659, Tiedemann et al. (1989) extended this conclusion back to $4 \mathrm{Ma}$, and showed that the ITCZ remained more or less stationary over this period. Other studies have, however, concluded that such N-S movements were possible during glacial/interglacial changes. Kolla et al. (1979) showed that geographic changes in the deposition area of eolian quartz in the equatorial Atlantic could indicate that an $8^{\circ}$ southward migration of the northerly belt of trade winds occurred at the LGM compared to today. This southward movement of the low-latitude, atmospheric system at the LGM is also suggested by Balsam et al. (1995). Models of hydrographic and atmospheric circulation clearly indicate that decreased SST in the North Atlantic during the LGM resulted in the southward shift of the limits of the subtropical gyre, leading for instance to the southward motion of the Gulf Stream (Keffer et al., 1988). Thus, although available paleoclimatic data appear to be contradictory, evidence exists that the glacial/interglacial $\mathrm{N}-\mathrm{S}$ migration of major atmospheric and hydrographic elements is possible and that a scenario that would imply modifications of the latitudinal structure of the thermocline topography is realistic.

\section{Trade-Wind Scenario vs. Latitudinal-Migration Scenario}

Our data show clearly that the initiation of large continental ice sheets at the "mid-Pleistocene revolution" probably had a major impact on surface circulation in the western equatorial Atlantic. Given the available data, however, it is not possible to unambiguously identify the respective role of the "trade-wind" (zonal) and the "latitudinal" scenarios in controlling the thermocline structure at the Ceara Rise around $1 \mathrm{Ma}$. As the trade-wind scenario seems to be unlikely before $960 \mathrm{ka}$ (see above), the important and rapid change observed in the phase relationships between $\delta^{18} \mathrm{O}$ and $F$. Profunda 41-k.y. oscillations may indicate that the western equatorial Atlantic experienced a drastic change from a latitudinal to a zonal control of the thermocline dynamics at the "mid-Pleistocene revolution." It seems unlikely, however, that the latitudinal scenario acting prior to $960 \mathrm{ka}$ would have no effect after $920 \mathrm{ka}$. We tend to favor, therefore, a second hypothesis in which the latitudinal mechanisms had always had the major impact in controlling the thermocline structure in the western equatorial Atlantic over the time period studied. In this hypothesis, the important changes observed at the "mid-Pleistocene revolution" would reflect a modification of the latitudinal structure of the thermocline due to a permanent shift of the southern limit of the subtropical gyre related to the development of large continental icesheets. If this scenario is correct, then we should expect that such a latitudinal shift of the western equatorial atmospheric and hydrographic circulation system would have major impacts on the crossequatorial transport of water to the north Atlantic.

\section{CONCLUSIONS}

Cross-equatorial transport of surface water in the western equatorial Atlantic is an important element of the conveyor belt return flow and contributes to the transport of heat and salt to the northern Atlantic Ocean. Our results show changes in the hydrographic conditions of the western equatorial Atlantic associated with major changes in Earth's climate at the "mid-Pleistocene revolution." Studying variations in the time domain from isolated stratigraphic records, however, cannot provide complete information about the exact mechanisms and origins of these low-latitude, surface hydrographic changes. It will be necessary to address these changes in the spatial domain by reconstructing paleomaps of the thermocline topography based on a latitudinal array of good sediment records.

\section{ACKNOWLEDGMENTS}

F.B. would like to thank the crew, technicians, and fellow scientific members of Leg 154 for an enjoyable, interesting, and productive experience. We are grateful to William F. Ruddiman and Will Howard for constructive criticisms that helped to improve the manuscript. Special thanks go to Sophie Bieda for carbonate data generation and sample preparation, and to Danielle Dole for isotopic analyses. This is contribution no. 1823 of the CFR, and no. 97001 of the LGQ. This work was supported by funding from INSU/CNRS (under DYTEC and IST programs).

\section{REFERENCES}

Balsam, W.L., Otto-Bliesner, B.L., and Deaton, B.C., 1995. Modern and last glacial maximum eolian sedimentation patterns in the Atlantic Ocean interpreted from sediment iron oxide content. Paleoceanography, 10:493-507.

Bassinot, F.C., Beaufort, L., Vincent, E., Labeyrie, L.D., Rostek, F., Müller, P.J., Quidelleur, X., and Lancelot, Y., 1994a. Coarse fraction fluctuations in pelagic carbonate sediments from the tropical Indian Ocean: a 1,500k.y.r record of carbonate dissolution. Paleoceanography, 9(4):579-600.

Bassinot, F., Labeyrie, L., Vincent, E., Quidelleur, X., Shackleton, N.J., and Lancelot, Y., 1994b. The astronomical theory of climate and the age of the Brunhes-Matuyama magnetic reversal. Earth Planet. Sci. Lett., 126:91-108.

Beaufort, L., 1994. Climatic importance of the modulation of the 100-k.y.r cycle inferred from 16 m.y. long Miocene records. Paleoceanography, 9:821-834.

Beaufort, L., and Aubry, M.-P., 1990. Fluctuations in the composition of Late Miocene calcareous nannofossil assemblages as a response to orbital forcing. Paleoceanography, 5:845-865.

Berger, A., and Loutre, M.F., 1991. Insolation values for the climate of the last 10 million years. Quat. Sci. Rev., 10:297-317.

1994. Precession, eccentricity, obliquity, insolation and paleoclimates. In Duplessy, J.-C. and Spyridakis, M.T. (Eds.), Long-Term Climatic Variations - Data and Modeling, NATO ASI Ser., 22:107-152.

Berger, W.H., Bonneau, M.-C., and Parker, F.L., 1982. Foraminifera on the deep-sea floor: lysocline and dissolution rate. Oceanol. Acta, 5:249-258.

Berger, W.H., Killingley, J.S., and Vincent, E., 1978. Stable isotopes in deepsea carbonates: Box cores ERDC-92, west equatorial Pacific. Oceanol. Acta, 1:203-216.

Berger, W.H., Yasuda, M.K., Bickert, T., Wefer, G., and Takayama, T., 1994. Quaternary time scale for the Ontong Java Plateau: Milankovitch template for Ocean Drilling Program Site 806. Geology, 22:463-467.

Broecker, W.S. and Takahashi, T., 1978. The relationship between lysocline depth and in-situ carbonate ion concentration. Deep Sea Res Part A., 25:65-95.

CLIMAP Project Members, 1981. Seasonal reconstructions of the earth's surface at the last glacial maximum. Geol. Soc. Am., Map and Chart Ser., MC36.

Curry, W.B. and Lohmann, G.P., 1990. Reconstructing past particles fluxes in the tropical Atlantic ocean. Paleoceanography, 5:487-505.

Curry, W.B., Shackleton, N.J., Richter, C., et al., 1995. Proc. ODP, Init. Repts., 154: College Station, TX (Ocean Drilling Program).

DeBlonde, G., and Peltier, W.R., 1991. A one-dimensional model of continental ice volume fluctuations through the Pleistocene: implications for the origin of the mid-Pleistocene climate transition. J. Clim., 4:318-344.

Defant, A., 1961. Physical Oceanography: Oxford (Pergamon Press).

deMenocal, P.B., 1995. Plio-Pleistocene African climate Science, 270:53-59.

deMenocal, P.B., and Rind, D., 1993. Sensitivity of Asian and African climate to variations in seasonal insolation, glacial ice cover, sea surface temperature, and Asian orography. J. Geophys. Res., 98(D4):7265-7287.

Duplessy, J.-C., Bé, A.W.H., and Blanc, P.L., 1981. Oxygen and carbon isotopic composition and biogeographic distribution of planktonic foraminifers in the Indian Ocean. Palaeogeogr., Palaeoclimatol., Palaeoecol., 33:9-46. 
Ghil, M., and Le Treut, H., 1981. A climate model with cryodynamics and geodynamics. J. Geophys. Res., 86:5262-5270.

Gordon, A.L., 1986. Interocean exchange of thermocline water. J. Geophys. Res., 91:5037-5046.

Hastenrath, S., 1994. Climate Dynamics of the Tropics: Dordrecht (Kluwer Academic).

Imbrie, J., Berger, A., Boyle, E.A., Clemens, S.C., Duffy, A., Howard, W.R., Kukla, G., Kutzbach, J., Martinson, D.G., McIntyre, A., Mix, A.C., Molfino, B., Morley, J.J., Peterson, L.C., Pisias, N.G., Prell, W.L., Raymo, M.E., Shackleton, N.J., and Toggweiler, J.R., 1993. On the structure and origin of major glaciation cycles. 2. The 100,000-year cycle. Paleoceanography, 8:699-735.

Imbrie, J. and Imbrie, J.Z., 1980. Modeling the climatic response to orbital variations. Science 207:943-953.

Keffer, T., Martinson, D.G., and Corliss, B.H., 1988. The position of the Gulf Stream during Quaternary glaciations. Science, 241:440-442.

Kolla, V., Biscaye, P.E., and Hanley, A.F., 1979. Distribution of quartz in Late Quaternary Atlantic sediments in relation to climate. Quat. Res., 11:261-277.

Lamb, P.J., 1981. Estimate of annual variation of Atlantic Ocean heat transport. Nature, 290:766-768.

Le, J., and Shackleton, N.J., 1992. Carbonate dissolution fluctuations in the western equatorial Pacific during the late Quaternary. Paleoceanography, $7: 21-42$.

Le Treut, H., and Ghil, M., 1983. Orbital forcing, climatic interactions, and glaciation cycles. J. Geophys. Res., 88:5167-5190.

Maasch, K.A., 1988. Statistical detection of the mid-Pleistocene transition. Clim. Dyn., 2:133-143.

Manabe, S., and Broccoli, A.J., 1985. The influence of continental ice sheets on the climate of an ice age. J. Geophys. Res., 90:2167-2190.

Martinson, D.G., Pisias, N.G., Hays, J.D., Imbrie, J., Moore, T.C., and Shackleton, N.J., 1987. Age dating and the orbital theory of the ice ages: development of a high-resolution 0 to 300,000 year chronostratigraphy. Quat. Res., 27:1-29.

McIntyre, A., Ruddiman, W.F., Karlin, K., and Mix, A.C., 1989. Surface water response of the equatorial Atlantic ocean to orbital forcing. Paleoceanography, 4:19-55.

Merle, J., 1980a. Seasonal heat-budget in the equatorial Atlantic Ocean. $J$. Phys. Oceanogr., 10:464-469.

, $1980 \mathrm{~b}$. Seasonal variation of heat-storage in the tropical Atlantic Ocean. Oceanol. Acta, 3:455-463.

Mienert, J., Curry, W.B., and Sarnthein, M., 1988. Sonostratigraphic records from equatorial Atlantic deep-sea carbonates: paleoceanographic and climatic relationships. Mar. Geol., 83:9-20.

Molfino, B. and McIntyre, A., 1990. Precessional forcing of nutricline dynamics in the Equatorial Atlantic. Science, 249:766-769.

Mosimann, J.E., 1965. Statistical methods for the pollen analyst: multinomial and negative multinomial techniques. In Kummel, B. and Raup, D. (Eds.), Handbook of Paleontological Techniques: San Francisco (W.H. Freeman), 636-673.

Okada, H. And Honjo, S., 1973. The distribution of oceanic coccolithophorids in the Pacific. Deep-Sea Res., 20:355-374.

Paillard, D., and Labeyrie, L.D., 1995. An optimal method to establish timescales using all available stratigraphic information: the software "analyseries." Vth International Conference on Paleoceanography (ICP V), 75. (Abstract)

Peterson, L.C., and Prell, W.L., 1985. Carbonate dissolution in recent sediments of the eastern equatorial Indian Ocean: preservation patterns and carbonate loss above the lysocline. Mar. Geol., 64:259-290.

Pisias, N.G. and Moore, T.C., 1981. The evolution of Pleistocene climate: A time series approach. Earth Planet. Sci. Lett., 52:450-458.

Raffi, I., Backman, J., Rio, D., and Shackleton, N., 1993. Plio-Pleistocene nannofossil biostratigraphy and calibration to oxygen isotope stratigra- phies from Deep Sea Drilling Project Site 607 and ODP Site 677, Paleoceanography, 8:387-408.

Raymo, M. E., Ruddiman, W.F., Shackleton, N.J., and Oppo, D.W., 1990. Evolution of Atlantic-Pacific $\delta^{13} \mathrm{C}$ gradients over the last 2.5 m.y. Earth Planet. Sci. Lett., 97:353-368.

Ruddiman, W.F., Cameron, D., and Clement, B.M., 1987. Sediment disturbance and correlation of offset holes drilled with the hydraulic piston corer: Leg 94. In Ruddiman, W.F., Kidd, R.B., Thomas, E. et al. (Eds.), Init. Repts. DSDP, 94 (Part 2): Washington (U.S. Govt. Printing Office), 615-634.

Ruddiman, W.F., and Kutzbach, J.E., 1989. Forcing of late Cenozoic Northern Hemisphere climate by plateau uplift in southern Asia and the American West. J. Geophys. Res., 94:18409-18427.

Ruddiman, W.F. and McIntyre, A., 1984. Ice-age thermal response and climatic role of the surface Atlantic ocean, $40^{\circ} \mathrm{N}$ to $63^{\circ} \mathrm{N}$. Geol. Soc. Am. Bull., 95:381-396.

Ruddiman, W.F., McIntyre, A., and Raymo, M., 1986. Paleo-environmental results from North Atlantic Sites 607 and 609. In Ruddiman, W.F., Kidd, R.B., Thomas, E., et al., Init. Repts. DSDP, 94 (Part 2): Washington (U.S. Govt. Printing Office), 855-878.

Ruddiman, W.F., Raymo, M.E., Martinson, D.G., Clement, B.M., and Backman, J., 1989. Pleistocene evolution: Northern hemisphere ice sheets and North Atlantic ocean. Paleoceanography, 4:353-412.

Ruddiman, W.F., Raymo, M., and McIntyre, A., 1986. Matuyama 41,000year cycles: North Atlantic Ocean and northern hemisphere ice sheets. Earth Planet. Sci. Lett., 80:117-129.

Saltzman, B., 1987. Carbon dioxide and the $\delta^{18} \mathrm{O}$ record of late-Quaternary climatic change: a global model. Climate Dynamics, 1:77-85.

Sarnthein, M., Tezlaff, G., Koopmann, B., Wolter, K., and Pflaumann, U., 1981. Glacial and interglacial wind regimes over the eastern subtropical Atlantic and Northwest Africa. Nature, 293:193-196.

Schmitz, W.J., Jr., and McCartney, M.S., 1993. On the north Atlantic circulation. Rev. Geophys., 31:29-49.

Shackleton, N.J., Berger, A., and Peltier, W.R., 1990. An alternative astronomical calibration of the lower Pleistocene timescale based on ODP Site 677. Trans. R. Soc. Edinburgh Earth Sci., 81:251-261.

Shackleton, N.J., and Opdyke, N.D., 1976. Oxygen isotope and paleomagnetic stratigraphy of Pacific Core V28-239: late Pliocene to latest Pleistocene. In Cline, R.M., and Hays, J.D. (Eds.), Investigations of Late Quaternary Paleoceanography and Paleoclimatology. Mem.-Geol. Soc. Am., 145:449-464.

Shipboard Scientific Party, 1995a. Site 925. In Curry, W.B., Shackleton, N.J., Richter, C., et al., Proc. ODP, Init. Repts., 154: College Station, TX (Ocean Drilling Program), 55-152.

, 1995b. Site 928. In Curry, W.B., Shackleton, N.J., Richter, C., et al., Proc. ODP, Init. Repts., 154: College Station, TX (Ocean Drilling Program), 281-336.

Thunell, R.C., 1982a. Carbonate dissolution and abyssal hydrography in the Atlantic Ocean. Mar. Geol., 47:165-180.

- $1982 \mathrm{~b}$. Optimum indices of calcium carbonate dissolution in deep-sea sediments. Geology, 4:525-528.

Tiedemann, R., Sarnthein, M., Shackleton, N.J., 1994. Astronomical timescale for the Pliocene Atlantic $\delta^{18} \mathrm{O}$ and dust flux records of Ocean Drilling Program Site 659. Paleoceanography, 9:619-638.

Tiedemann, R., Sarnthein, M., and Stein, R., 1989. Climatic changes in the western Sahara: aeolo-marine sediment record of the last 8 million years (Sites 657-661). In Ruddiman, W., Sarnthein, M., et al., Proc. ODP, Sci. Results, 108: College Station, TX (Ocean Drilling Program), 241-277.

Date of initial receipt: 16 January 1996

Date of acceptance: 6 August 1996

Ms 154SR-108 